TRANSACTIONS OF THE

AMERICAN MATHEMATICAL SOCIETY

Volume 348, Number 7, July 1996

\title{
ON GELFAND-KIRILLOV TRANSCENDENCE DEGREE
}

\author{
JAMES J. ZHANG
}

\begin{abstract}
We study some basic properties of the Gelfand-Kirillov transcendence degree and compute the transcendence degree of various infinite-dimensional division algebras including quotient division algebras of quantized algebras related to quantum groups, 3-dimensional Artin-Schelter regular algebras and the 4-dimensional Sklyanin algebra.
\end{abstract}

\section{INTRODUCTION}

The Gelfand-Kirillov transcendence degree was introduced in [GK] by I. M. Gelfand and A. A. Kirillov in order to show that the $n$-th and $m$-th Weyl division algebras $D_{n}$ and $D_{m}$ are not isomorphic if $n \neq m$. For an algebra $A$ over a base field $k$, the Gelfand-Kirillov transcendence degree of $A$ is defined to be

$$
\operatorname{Tdeg}(A)=\sup _{V} \inf _{b} \varlimsup_{n \rightarrow \infty} \log _{n} \operatorname{dim}\left((k+b V)^{n}\right)
$$

where $V$ ranges over the subframes (i.e. finite dimensional subspaces containing 1) of $A$ and $b$ ranges over the regular elements of $A$. The dimension of a $k$-vector space is always denoted by dim. The Gelfand-Kirillov dimension of $A$, which was also introduced in [GK], is defined to be

$$
\operatorname{GKdim}(A)=\sup _{V} \varlimsup_{n \rightarrow \infty} \log _{n} \operatorname{dim}\left(V^{n}\right)
$$

where $V$ ranges over the subframes of $A$. Gelfand and Kirillov proved that GKdim and Tdeg of the $n$-th Weyl algebra $A_{n}$ are $2 n$ and that Tdeg of the quotient division algebra $D_{n}$ of $A_{n}$ is $2 n$.

On the other hand, L. Makar-Limanov showed that the first Weyl division algebra $D_{1}$ (and hence $D_{n}$ for all $n \geq 1$ ) contains a noncommutative free subalgebra of two variables [M-L]. This implies that $\operatorname{GKdim}\left(D_{n}\right)=\infty$ for all $n \geq 1$ [KL, 8.18]. Hence GKdim is not a good invariant for these division algebras, and indeed it is not a good invariant for many other non-PI division algebras.

Since the 1980's several new examples of noncommutative algebras have been discovered. In particular, quantum groups and related algebras have been studied extensively. Hence a familiar question arises: How to determine whether two quotient division algebras of quantized algebras are isomorphic or not. This question

Received by the editors May 9, 1995.

1991 Mathematics Subject Classification. Primary 16P90, 12E15, 16K40, 16 S80.

Key words and phrases. Gelfand-Kirillov transcendence degree, Gelfand-Kirillov dimension, division algebra, noncommutative domain.

This research was supported in part by the NSF.

(C)1996 American Mathematical Society 
can be solved partially if there is a good invariant for division algebras. One aim of this paper is to show that the Gelfand-Kirillov transcendence degree is a good invariant for division algebras of quantized algebras (see sections $6,7,10$ ).

If $A$ is a commutative field, then $\operatorname{Tdeg}(A)$ is equal to the (classical) transcendence degree $\operatorname{tdeg}(A)$. This is of course a justification of calling Tdeg a transcendence degree. If $A$ is a noncommutative division algebra, it is difficult to compute $\operatorname{Tdeg}(A)$ because it is not easy to determine $\operatorname{dim}\left((k+b V)^{n}\right)$ for all different choices of $b, V$, and $n$. W. Borho and H. Kraft in [BK] calculated Tdeg for the quotient division algebras of almost commutative domains (for example, universal enveloping algebras of finite dimensional Lie algebras) and M. Lorenz in [Lo] calculated Tdeg for the quotient division algebras of some (twisted) group algebras. Not much else was known about the Gelfand-Kirillov transcendence degree. Another aim of this paper is to discuss basic properties of Tdeg, especially to study some properties which were not discussed in [GK], [BK] and [Lo] (see sections 3, 4, 5, 9).

Most non-PI division algebras in which we are interested are quotient division algebras of some noetherian domains of finite GKdim. Hence it will be helpful if we can find a close relationship between Tdeg of a division algebra and GKdim of its subalgebra. Thus we introduce the following notion. An algebra $A$ with total quotient algebra $Q(A)$ is said to be Tdeg-stable if $\operatorname{Tdeg}(Q(A))=\operatorname{Tdeg}(A)=$ $\operatorname{GKdim}(A)$. Gelfand and Kirillov proved that the Weyl algebras are Tdeg-stable because

$$
\operatorname{Tdeg}\left(D_{n}\right)=\operatorname{Tdeg}\left(A_{n}\right)=\operatorname{GKdim}\left(A_{n}\right)=2 n .
$$

Tdeg-stable is a technical but useful notion. If $A$ is Tdeg-stable, one can compute $\operatorname{Tdeg}(A)$ and $\operatorname{Tdeg}(Q(A))$ by only determining $\operatorname{GKdim}(A)$. If $A$ is not Tdeg-stable, one should try to find a subalgebra $B \subset A$ such that (a) $B$ is Tdeg-stable and (b) $A$ is between $B$ and $Q(B)$. If this is the case, it is easy to show $\operatorname{Tdeg}(Q(A))=$ $\operatorname{Tdeg}(A)=\operatorname{GKdim}(B)$. Hence in many cases we reduce a problem of calculating $\operatorname{Tdeg}(Q)$ for a division algebra $Q$ to a problem of calculating $\operatorname{GKdim}(B)$ for some noetherian subalgebra $B \subset Q$. Therefore one of our basic questions is to determine which algebras are Tdeg-stable.

Theorem 1.1. The following algebras are Tdeg-stable.

(1) Semiprime Goldie algebras with GKdim at most 2.

(2) Semiprime Goldie PI algebras.

(3) Skew polynomial algebras $k_{p_{i j}}\left[x_{1}, \cdots, x_{n}\right]$ and $k_{p_{i j}}\left[x_{1}, x_{1}^{-1}, \cdots, x_{n}, x_{n}^{-1}\right][L o$, 2.2].

(4) Universal enveloping algebras $U(L)$ of finite dimensional Lie algebras $L$ [BK, 6.5].

(5) The Weyl algebras $A_{n}[G K, 6]$.

(6) Quantum matrix algebras $M_{q, p_{i j}}(n)$ and $G L_{q, p_{i j}}(n)$.

(7) Quantum Weyl algebras $A_{n}\left(q, p_{i j}\right)$.

(8) Quantum universal enveloping algebra $U_{q}\left(s l_{2}\right)$.

(9) $A[x]$ and $A\left[x, x^{-1}\right]$ if $A$ is Tdeg-stable.

(10) 3-dimensional Artin-Schelter regular algebras generated in degree 1.

(11) The 4-dimensional Sklyanin algebra.

There are other Tdeg-stable algebras which are described in sections 5, 9 and 10. Given a division algebra $Q$, it is unknown if there exists a Tdeg-stable subalgebra $B$ of $Q$ such that $Q(B)=Q$ (although that is true in many cases). However there 
exists a subalgebra $B$ such that $Q(B)=Q$ and the difference between $\operatorname{GKdim}(B)$ and $\operatorname{Tdeg}(Q)$ can be arbitrary small. The precise statement is the following.

Theorem 1.2. Let $A$ be a domain and suppose that the quotient division algebra $Q(A)$ is finitely generated as a division algebra.

(1) $\operatorname{Tdeg}(A)=\inf \operatorname{GKdim}(B)$ where $B$ ranges over all finitely generated subalgebras of $A$ such that $Q(B)=Q(A)$.

(2) If $V$ is a subframe of $A$ such that $Q(k[V])=Q(A)$, then

$$
\operatorname{Tdeg}(A)=\inf _{b \in A-\{0\}} \operatorname{GKdim}(k[b V]) .
$$

If $A$ is a finitely generated domain, then Theorem 1.2(2) provides a simplification of the definition of Tdeg. As a consequence of Theorem 1.2(1), if $A$ is a finitely generated and Tdeg-stable domain, then $\operatorname{GKdim}(A)=\operatorname{GKdim}(B)$ for every subalgebra $B$ such that $Q(B)=Q(A)$. For example, let $A_{n}$ be the $n$-th Weyl algebra generated by $\left\{x_{1}, \ldots, x_{n}\right\} \cup\left\{\partial_{1}, \cdots, \partial_{n}\right\}$. Then each subalgebra generated by $\left\{b, b x_{1}, \cdots, b x_{n}, b \partial_{1}, \cdots, b \partial_{n}\right\}$ has GKdim $2 n$ for every nonzero $b \in A_{n}$.

Recently M. Artin and J. T. Stafford classified connected graded domains of GKdim 2 in term of projective curves [ASta]. The first step of their work was to prove the following result [ASta, 0.1(i)].

Theorem (Artin-Stafford). Let $A$ be a finitely generated connected $\mathbb{N}$-graded domain of GKdim 2, with graded quotient algebra $D\left[z, z^{-1}, \sigma\right]$. Then $D$ is a finite module over its center $F$, and $F$ is a finitely generated field extension of $k$ of transcendence degree one.

In this case $D$ is a PI division algebra and hence $\operatorname{GKdim}(D)=\operatorname{tdeg}(D)=1$. It is natural to ask if a similar result holds for connected graded domains of GKdim 3. If $A$ is a connected graded domain with finite GKdim, then $A$ is graded Goldie and the graded quotient algebra $Q_{g r}(A)$ of $A$ is isomorphic to $D\left[z, z^{-1}, \sigma\right]$, where $D$ is a division algebra and $\sigma$ is an automorphism of $D$. If $\operatorname{GKdim}(A)=3$, then in general $D$ is not a PI division algebra and in many cases $\operatorname{GKdim}(D)=\infty$. Hence the classical transcendence degree is not suitable for $D$. One reasonable conjecture is the following.

Conjecture. Let $A$ be a finitely generated connected graded domain of GKdim 3, with graded quotient algebra $D\left[z, z^{-1}, \sigma\right]$. Then $\operatorname{Tdeg}(D)=2$.

If $\operatorname{GKdim}(A) \geq 4$, then the conjecture should be $\operatorname{Tdeg}(D) \leq \operatorname{GKdim}(A)-1$. There is a finitely generated, connected graded and Tdeg-stable domain of GKdim 4 , with graded quotient algebra $D\left[z, z^{-1}, \sigma\right]$, such that $D$ is a field of GKdim 2 [Example 9.8]. We will see that $\operatorname{Tdeg}(D)=1$ if and only if $\operatorname{GKdim}(D)=1$ [Theorem 4.3]. Hence [ASta, 1.2] implies that $\operatorname{Tdeg}(D) \geq 2$ if $\operatorname{GKdim}(A)=3$. The following partial result will be proved, but the conjecture remains open in general.

Theorem 1.3. Let $A$ be a connected graded right noetherian domain of GKdim 3 , with graded quotient algebra $D\left[z, z^{-1}, \sigma\right]$. If $A$ contains a normal element of positive degree, then $\operatorname{Tdeg}(D)=2$.

By the classification of $[\mathrm{AS}]$ and [ATV1,2], every 3-dimensional Artin-Schelter regular algebra generated in degree 1 contains a normal element of positive degree. Hence Theorem 1.3 applies to these algebras. There are many unsolved (but very 
basic) questions about Tdeg. For example, it is unknown if $\operatorname{Tdeg}\left(D\left[z, z^{-1}, \sigma\right]\right) \geq$ $\operatorname{Tdeg}(D)+1$ holds. Other unsolved questions can be found in $[\mathrm{Sc}]$.

The author would like to thank Paul Smith for useful conversations and helpful comments on the subject.

\section{BAsic PRoperties}

In this section we investigate some elementary properties of the Gelfand-Kirillov transcendence degree. Let $k$ be a field. Throughout the paper, vector space, algebra, Gelfand-Kirillov dimension, Gelfand-Kirillov transcendence degree (and other notions) are defined over the ground field $k$ unless indicated otherwise. We will use basic properties of the Gelfand-Kirillov dimension frequently, which can be found in [KL], [NV2, Ch. 10] and [MR, Ch. 8]. Recall that $V$ is called a subframe of $A$ if $V$ is a finite dimensional subspace of $A$ containing the identity 1 . If $A$ is generated by a subframe $V$, then by $[\mathrm{KL}, 1.1]$

$$
\operatorname{GKdim}(A)=\varlimsup_{n \rightarrow \infty} \log _{n}\left(\operatorname{dim}\left(V^{n}\right)\right) .
$$

For an algebra $A$, the Gelfand-Kirillov transcendence degree, denoted by Tdeg $(A)$, is defined to be

$$
\operatorname{Tdeg}(A)=\sup _{V} \inf _{b} \operatorname{GKdim}(k[b V])
$$

where $V$ ranges over the subframes of $A$ and $b$ ranges over the regular elements of $A$ (see $[N V 2$, p. 330]). Since $\operatorname{GKdim}(k[b V]) \leq \operatorname{GKdim}(A)$, the next proposition is clear.

Proposition 2.1. Let $A$ be a k-algebra. Then $\operatorname{Tdeg}(A) \leq \operatorname{GKdim}(A)$.

Proposition 2.2. If $A$ is commutative, then $\operatorname{Tdeg}(A)=\operatorname{GKdim}(A)$. As a consequence, if $A$ is a commutative field, then $\operatorname{Tdeg}(A)=\operatorname{GKdim}(A)=\operatorname{tdeg}(A)$.

Recall that tdeg denotes the (classical) transcendence degree, and $\operatorname{GKdim}(A)=$ $\operatorname{tdeg}(A)$ if $A$ is a commutative field [NV2, 10.3.3.1].

Proof. By definition we have

$$
\operatorname{Tdeg}(A)=\sup _{V} \inf _{b} \operatorname{GKdim}(k[b V])
$$

and

$$
\operatorname{GKdim}(A)=\sup _{V} \operatorname{GKdim}(k[V]) .
$$

It suffices to show that $\operatorname{GKdim}(k[b V]) \geq \operatorname{GKdim}(k[V])$ for all regular elements $b$ of $A$. Since $A$ is commutative, we have

$$
\operatorname{dim}\left((k+b V)^{n}\right) \geq \operatorname{dim}\left((b V)^{n}\right)=\operatorname{dim}\left(b^{n} V^{n}\right)=\operatorname{dim}\left(V^{n}\right) .
$$

Hence $\operatorname{GKdim}(k[b V]) \geq \operatorname{GKdim}(k[V])$. 
Example 2.3. There are algebras $A$ such that $\operatorname{Tdeg}(A)<\operatorname{GKdim}(A)<\infty$.

(1) Lorenz's example [Lo, 2.5]. Let $R$ be the commutative domain $k\left[x, x^{-1}, y, y^{-1}\right]$ and let $\sigma$ be the automorphism defined by $\sigma(x)=x$ and $\sigma(y)=y x$. Then $S=R\left[z, z^{-1}, \sigma\right]$ is isomorphic to the group algebra $k[G]$ where $G=\langle y, z| x=$ $[y, z]$ central $\rangle$. By $[\operatorname{Lo}, 2.5$ and 2.2] $\operatorname{GKdim}(S)=\operatorname{Tdeg}(S)=4$. Let $\mathcal{C}$ be the multiplicatively closed set generated by the elements $\left\{1-y x^{m} \mid m \in \mathbb{Z}\right\}$. Then $T=R_{\mathcal{C}}\left[z, z^{-1}, \sigma\right]$ is a noetherian domain and it is a localization of $S$. By [Lo, 2.4 and 2.5], $5 \leq \operatorname{GKdim}(T) \leq 8$. By Proposition 3.1(3) in $\operatorname{Section} 3, \operatorname{Tdeg}(T) \leq 4$. Therefore $\operatorname{Tdeg}(T)<\operatorname{GKdim}(T) \leq 8$.

Another property of the algebra $S=R\left[z, z^{-1}, \sigma\right]$ is

$$
\operatorname{Tdeg}\left(R\left[z, z^{-1}, \sigma\right]\right)=4>\operatorname{Tdeg}(R)+1
$$

where $\operatorname{Tdeg}(R)=\operatorname{GKdim}(R)=2$ by Proposition 2.2 .

(2) A. Joseph's example [Jo]. Let $A_{1}$ be the first Weyl algebra generated by $x, \partial$ subject to the relation $\partial x-x \partial=1$ and $S$ the multiplicatively closed set generated by the elements $\{x \partial-m \mid m \in \mathbb{Z}\}$. By [KL, 4.11], $S$ is an Ore set and $\operatorname{GKdim}\left(S^{-1} A_{1}\right)=3$. On the other hand, we will see that $\operatorname{Tdeg}\left(S^{-1} A_{1}\right)=2$ at the end of Section 3. Hence $\operatorname{Tdeg}\left(S^{-1} A_{1}\right)<\operatorname{GKdim}\left(S^{-1} A_{1}\right)$.

G. Bergman proved that no number in the open interval $(1,2)$ appears as the GKdim of an algebra [Be]. It is easy to see that no GKdim numbers are in the open interval $(0,1)$. By the definition of Tdeg (see (2.0)), we have the following.

Proposition 2.4. Let $A$ be an algebra. Then $\operatorname{Tdeg}(A)$ is either 0,1 , or $\geq 2$.

It is unknown if there is a division algebra $A$ such that $\operatorname{Tdeg}(A)$ is a non-integer. For convenience, we denote, for every subframe $V$ of $A$,

$$
I_{V}(A)=\inf _{b} \operatorname{GKdim}(k[b V])
$$

where $b$ ranges over all regular elements of $A$. The following lemma is clear.

Lemma 2.5. Let $A$ be an algebra and let $V$ and $V^{\prime}$ be subframes of $A$.

(1) If $V \subset V^{\prime}$, then $I_{V}(A) \leq I_{V^{\prime}}(A)$.

(2) Suppose $\Phi=\left\{V^{\prime}\right\}$ is a set of subframes of $A$ which is cofinal amongst all subframes of $A$, namely, every subframe $V$ of $A$ is contained in a subframe $V^{\prime}$ in this particular set $\Phi$. Then $\operatorname{Tdeg}(A)=\sup _{V^{\prime} \in \Phi} I_{V^{\prime}}(A)$.

Proposition 2.6. Let $B$ and $C$ be algebras and let $A=B \oplus C$. Then

$$
\operatorname{Tdeg}(A)=\max \{\operatorname{Tdeg}(B), \operatorname{Tdeg}(C)\} .
$$

Proof. Every regular element $a$ of $A$ is of form $b+c$ where $b$ and $c$ are regular elements of $B$ and $C$ respectively. For any subframes $V_{1}$ of $B$ and $V_{2}$ of $C$, we define a subframe $V^{\prime}$ of $A$ by $V^{\prime}=V_{1} \oplus V_{2}$. It is easy to see that the algebras $k\left[a V^{\prime}\right]$ and $k\left[b V_{1}\right] \oplus k\left[c V_{2}\right]$ differ by a 1-dimensional vector space. For every subframe $V$ of $A$, there is a subframe $V^{\prime}=V_{1} \oplus V_{2}$ such that $V \subset V^{\prime}$. By Lemma 2.5(2), to compute $\operatorname{Tdeg}(A)$ we only need to take the supremum of $I_{V^{\prime}}(A)$ over all $V^{\prime}=V_{1} \oplus V_{2}$. Hence

$$
\begin{aligned}
& \max \{\operatorname{Tdeg}(B), \operatorname{Tdeg}(C)\}=\max \left\{\sup _{V_{1}} \inf _{b} \operatorname{GKdim}\left(k\left[b V_{1}\right]\right), \sup _{V_{2}} \inf _{c} \operatorname{GKdim}\left(k\left[c V_{2}\right]\right)\right\} \\
& =\sup _{V_{1}, V_{2}} \inf _{b, c} \max \left\{\operatorname{GKdim}\left(k\left[b V_{1}\right]\right), \operatorname{GKdim}\left(k\left[c V_{2}\right]\right)\right\} \\
& =\sup _{V^{\prime}=V_{1} \oplus V_{2}} \inf _{a=b+c} \operatorname{GKdim}\left(k\left[a V^{\prime}\right]\right)=\operatorname{Tdeg}(A) .
\end{aligned}
$$


Proposition 2.7. Let $A$ be an algebra and let $M_{n}(A)$ be the $n \times n$ matrix algebra over $A$.

(1) $\operatorname{Tdeg}(A) \geq \operatorname{Tdeg}\left(M_{n}(A)\right)$.

(2) If $A$ is a domain, then $\operatorname{Tdeg}(A)=\operatorname{Tdeg}\left(M_{n}(A)\right)$.

Proof. (1) For every subframe $V$ of $M_{n}(A)$, define $V^{\prime}=\sum_{i, j} k e_{i j}+\sum_{i, j, l, m} e_{i j} V e_{l m}$ where $\left\{e_{i j} \mid 1 \leq i, j \leq n\right\}$ are the matrix units of $M_{n}(A)$. Then $V^{\prime}$ is finite dimensional and $\left\{V^{\prime}\right\}$ is cofinal amongst all subframes of $M_{n}(A)$. For every such subframe $V^{\prime}$, there are $v_{l} \in A$ such that $V^{\prime}=\bigoplus_{i, j, l} k e_{i j} v_{l}$. Hence $V^{\prime}=M_{n}\left(V_{0}\right)$ where $V_{0}=\bigoplus_{l} k v_{l}$.

Let $b$ be a regular element of $A$. Then $b I_{n}$ is a regular element of $M_{n}(A)$. It is easy to see that the algebras $k\left[b I_{n} V^{\prime}\right]$ and $M_{n}\left(k\left[b V_{0}\right]\right)$ differ by a finite dimensional vector space. Indeed, as vector spaces, $M_{n}\left(k\left[b V_{0}\right]\right) / k\left[b I_{n} V^{\prime}\right] \cong M_{n}(k) / k$. Hence

$$
\begin{aligned}
I_{V}\left(M_{n}(A)\right) & \leq I_{V^{\prime}}\left(M_{n}(A)\right)=\inf _{a \in M_{n}(A)} \operatorname{GKdim}\left(k\left[a V^{\prime}\right]\right) \\
& \leq \inf _{b I_{n}} \operatorname{GKdim}\left(k\left[b I_{n} V^{\prime}\right]\right)=\inf _{b} \operatorname{GKdim}\left(M_{n}\left(k\left[b V_{0}\right]\right)\right) \\
& =\inf _{b} \operatorname{GKdim}\left(k\left[b V_{0}\right]\right)=I_{V_{0}}(A) .
\end{aligned}
$$

Since $V$ is arbitrary, $\operatorname{Tdeg}\left(M_{n}(A)\right) \leq \operatorname{Tdeg}(A)$.

(2) Suppose $A$ is a domain. Again we compare $I_{V^{\prime}}\left(M_{n}(A)\right)$ and $I_{V_{0}}(A)$. For every regular element $a \in M_{n}(A), a=\sum_{t, w} e_{t w} b_{t w}$ for $b_{t w} \in A$ and some $b_{i j} \neq 0$. Then

$$
a V^{\prime} \supset a V^{\prime} e_{j i} \supset k\left(\sum_{t} e_{t i} b_{t j}\right)+\sum_{l} k\left(\sum_{t} e_{t i} b_{t j}\right) v_{l} .
$$

It is easy to see that the map $f \longmapsto e_{i i} f$ defines an algebra epimorphism from the subalgebra generated by $k\left(\sum_{t} e_{t i} b_{t j}\right)+\sum_{l} k\left(\sum_{t} e_{t i} b_{t j}\right) v_{l}$ to the subalgebra $k\left[b_{i j} V_{0}\right]$. Therefore

$$
\operatorname{GKdim}\left(k\left[a V^{\prime}\right]\right) \geq \operatorname{GKdim}\left(k\left[b_{i j} V_{0}\right]\right) \geq I_{V_{0}}(A) .
$$

This implies that $I_{V^{\prime}}\left(M_{n}(A)\right) \geq I_{V_{0}}(A)$. By Lemma 2.5(2),

$$
\operatorname{Tdeg}\left(M_{n}(A)\right)=\sup _{V^{\prime}} I_{V^{\prime}}\left(M_{n}(A)\right) \geq \sup _{V_{0}} I_{V_{0}}(A)=\operatorname{Tdeg}(A) .
$$

If $A$ is not a domain, then the method used in the proof of Proposition 2.7(2) fails because $a=\sum e_{i j} b_{i j}$ may be regular even though none of the $b_{i j}$ is regular in $A$. An easy example is $A=k \oplus k=k e_{1} \oplus k e_{2}$. Then $a=\left(\begin{array}{ll}e_{1} & e_{2} \\ e_{2} & e_{1}\end{array}\right)$ is a regular element of $M_{2}(A)$ but $e_{1}$ and $e_{2}$ are not regular in $A$. On the other hand, no examples are known where $\operatorname{Tdeg}(A)>\operatorname{Tdeg}\left(M_{n}(A)\right)$. Another basic property of Tdeg is its symmetry.

Proposition 2.8. Let $A$ be an algebra. Then $\operatorname{Tdeg}(A)=\sup _{V} \inf _{b} \operatorname{GKdim}(k[V b])$. As a consequence, $\operatorname{Tdeg}(A)=\operatorname{Tdeg}\left(A^{\text {op }}\right)$.

Proof. It is suffices to show $k[b V] \cong k[V b]$ for every regular element $b$ and every subframe $V$. Suppose that $\left\{1, v_{1}, \cdots, v_{n}\right\}$ is a basis of $V$. Let $f\left(x_{0}, x_{1}, \cdots, x_{n}\right)$ be any noncommutative polynomial. Then $f_{l}=f\left(b, b v_{1}, \cdots, b v_{n}\right)$ is an element in 
$k[b V]$ and $f_{r}=f\left(b, v_{1} b, \cdots, v_{n} b\right)$ is an element in $k[V b]$. It is easy to check that $b f_{r}=f_{l} b$. Since $b$ is regular, $f_{l}=0$ if and only if $f_{r}=0$. Hence $f_{l} \longmapsto f_{r}$ is an isomorphism from $k[b V]$ to $k[V b]$.

Let us now state the Gelfand-Kirillov theorem for the Weyl division algebras. A proof will be given in Corollary 6.9.

Theorem 2.9 (Gelfand-Kirillov). Let $D_{n}$ be the $n$-th Weyl division algebra. Then $\operatorname{Tdeg}\left(D_{n}\right)=2 n$.

One basic property of classical transcendence degree is that $\operatorname{tdeg}(F) \leq \operatorname{tdeg}(D)$ if $F$ is a subfield of a commutative field $D$. However it is unknown whether $\operatorname{Tdeg}(B) \leq \operatorname{Tdeg}(D)$ holds for every division subalgebra $B$ of a noncommutative division algebra $D$. The following example shows that a division algebra of finite Tdeg may contain a subalgebra of infinite Tdeg.

Example 2.10. There is a division algebra $D$ and a subalgebra $B$ such that $\operatorname{Tdeg}(D)=2$ and $\operatorname{Tdeg}(B)=\infty$. As a consequence $\operatorname{GKdim}(D)=\operatorname{GKdim}(B)=\infty$.

Makar-Limanov's Example [M-L]. By Theorem 2.9, $\operatorname{Tdeg}\left(D_{1}\right)=2$. By [ML] $D_{1}$ contains a free subalgebra of two variables. $\operatorname{But} \operatorname{Tdeg}(k\langle x, y\rangle)=\infty$. To see this, let $V=k+k x+k y$. For every $b \in k\langle x, y\rangle, k[b V] \supset k[b x, b y]$. Since $k\langle x, y\rangle x \cap k\langle x, y\rangle y=0, k\langle x, y\rangle b x \cap k\langle x, y\rangle b y=0$. Hence $k[b x, b y]$ is isomorphic to a free algebra of two variables, and then $\operatorname{GKdim}(k[b V])=\infty$. Therefore $I_{V}(k\langle x, y\rangle)=$ $\infty$ and $\operatorname{Tdeg}(k\langle x, y\rangle)=\infty$.

\section{LocALization}

In this section we will show that $\operatorname{Tdeg}\left(S^{-1} A\right) \leq \operatorname{Tdeg}(A)$ for every algebra $A$ and every Ore set $S$ of regular elements of $A$. This inequality with Proposition 2.1 implies that the Weyl division algebras (and many other noncommutative division algebras) have finite Tdeg. Recall that for the Gelfand-Kirillov dimension we have the inequality $\operatorname{GKdim}\left(S^{-1} A\right) \geq \operatorname{GKdim}(A)$, and the equality will fail in general. By using the inequality for Tdeg, the definition of Tdeg can be simplified for finitely generated domains (compare (2.0) and (3.3.1)). At the end of the section we introduce the notion of Tdeg-stable and calculate Tdeg of the algebra $S^{-1} A_{1}$ in Example 2.3(2). For definitions of left and/or right Ore conditions, Ore domain and localization see for example [KL, Ch. 4] and [MR, Ch. 2].

Proposition 3.1. Let $A$ be an algebra and let $S$ be an Ore set of regular elements of $A$. Let $S^{-1} A$ be the localization of $A$ over $S$.

(1) Suppose $B$ is a subalgebra of $S^{-1} A$ containing $A$. Then

$$
\operatorname{Tdeg}(B)=\sup _{V_{0} \subset A} I_{V_{0}}(B)
$$

where $V_{0}$ ranges over all subframes of $A$.

(2) Let $B_{1} \subset B_{2}$ be subalgebras of $S^{-1} A$ containing $A$. Then $\operatorname{Tdeg}\left(B_{2}\right) \leq$ $\operatorname{Tdeg}\left(B_{1}\right)$.

(3) If $A \subset B \subset S^{-1} A, \operatorname{Tdeg}\left(S^{-1} A\right) \leq \operatorname{Tdeg}(B) \leq \operatorname{Tdeg}(A)$.

Proof. (1) For every subframe $V$ of $B$, there is an $s \in S$ such that $V^{\prime}:=s V$ is a subspace of $A$. Then $V \subset s^{-1}\left(V^{\prime}+k\right)$. For every regular element $r$ of $B, r s$ is a 
regular element of $B$. Hence

$$
I_{V}(B)=\inf _{b} \operatorname{GKdim}(k[b V]) \leq \inf _{r s} \operatorname{GKdim}\left(k\left[r s s^{-1}\left(V^{\prime}+k\right)\right]\right)=I_{V^{\prime}+k}(B) .
$$

Since $V$ is arbitrary and $V^{\prime}+k \subset A, \operatorname{Tdeg}(B)=\sup _{V_{0} \subset A} I_{V_{0}}(B)$.

(2) Let $V_{0}$ be a subframe of $A$. By assumption it is easy to see that every regular element of $B_{1}$ is a regular element of $B_{2}$. Then $I_{V_{0}}\left(B_{2}\right) \leq I_{V_{0}}\left(B_{1}\right)$. Hence the statement follows from (1). (3) is a consequence of (2).

If the set $S$ of all regular elements of $A$ is left and right Ore, then the total quotient algebra $S^{-1} A\left(=A S^{-1}\right)$ is denoted by $Q(A)$. By Proposition 3.1(3), $\operatorname{Tdeg}(Q(A)) \leq \operatorname{Tdeg}(A)$. The following is an analogue of A. V. Jategaonkar's result $[\mathrm{Ja}]$.

Proposition 3.2. If $A$ is a domain and $\operatorname{Tdeg}(A)<\infty$, then $A$ is a left and right Ore domain. Let $Q(A)$ be the quotient algebra of $A$. Then $\operatorname{Tdeg}(Q(A)) \leq \operatorname{Tdeg}(A)$.

Proof. It remains to prove the first assertion. If $A$ is not left Ore domain, then there exist $x, y \in A$ such that $A x \cap A y=0$. Then for every $b \in A, k[b x, b y]$ is isomorphic to the free algebra of two variables. Hence $I_{k+k x+k y}(A)=\infty$ and $\operatorname{Tdeg}(A)=\infty$. Contradiction. Therefore $A$ is left Ore. By Proposition 2.8, $A$ is also right Ore.

As a consequence of Proposition 3.2, if a domain $A$ is not left Ore then $\operatorname{Tdeg}(A)=$ $\infty$. Note that the right Ore condition does not imply the left Ore condition [Co, 1.3.1]. A division algebra is said to be finitely generated if it is a quotient algebra of a finitely generated algebra.

Theorem 3.3. Let $A$ be a domain and suppose that the quotient division algebra $Q(A)$ is finitely generated. Then

(1) $\operatorname{Tdeg}(A)=\inf \operatorname{GKdim}(B)$, where $B$ ranges over all finitely generated subalgebras of $A$ such that $Q(B)=Q(A)$.

(2) If $V$ is a subframe of $A$ with $Q(k[V])=Q(A)$, then $\operatorname{Tdeg}(A)=I_{V}(A)$.

Proof. (1) Since $B \subset A \subset Q(B)$, by Proposition 3.1(3), $\operatorname{Tdeg}(A) \leq \operatorname{Tdeg}(B) \leq$ $\operatorname{GKdim}(B)$. Hence $\mathrm{Tdeg}(A) \leq \inf \operatorname{GKdim}(B)$.

Conversely, we need to show $\operatorname{Tdeg}(A) \geq \inf \operatorname{GKdim}(B)$. We may assume that $\operatorname{Tdeg}(A)$ is finite. By the hypothesis there is a subframe $W$ such that $Q(A)=$ $Q(k[W])$. Since $W$ is finite dimensional, there is a subframe $V_{0}$ of $A$ such that every element in $W$ is a (non-commutative) rational function of elements in $V_{0}$. By definition

$$
I_{V_{0}}(A)=\inf _{b} \operatorname{GKdim}\left(k\left[b V_{0}\right]\right) \leq \operatorname{Tdeg}(A)<\infty .
$$

By Propositions 2.1 and 3.2, $\operatorname{GKdim}\left(k\left[b V_{0}\right]\right)<\infty$ implies that $k\left[b V_{0}\right]$ is left and right Ore. In this case it is clear that $Q\left(k\left[b V_{0}\right]\right) \supset V_{0}$, and then $Q\left(k\left[b V_{0}\right]\right)=$ $Q(k[W])=Q(A)$. Therefore

$$
\operatorname{Tdeg}(A) \geq I_{V_{0}}(A)=\inf \operatorname{GKdim}\left(k\left[b V_{0}\right]\right) \geq \inf \operatorname{GKdim}(B) .
$$

(2) Let $V$ be a subframe of $A$ such that $Q(k[V])=Q(A)$. For every nonzero element $b$ we have $Q(k[b V])=Q(k[V])=Q(A)$ if $\operatorname{GKdim}(k[b V])<\infty$. Therefore

$$
\operatorname{Tdeg}(A) \geq I_{V}(A)=\inf \operatorname{GKdim}(k[b V]) \geq \inf \operatorname{GKdim}(B)=\operatorname{Tdeg}(A)
$$

and thus $\operatorname{Tdeg}(A)=I_{V}(A)$. 
By Theorem 3.3(2), if $A$ is a domain finitely generated by a subframe $V$, then

$$
\operatorname{Tdeg}(A)=I_{V}(A)=\inf _{b} \operatorname{GKdim}(k[b V])
$$

where $b$ ranges over all nonzero elements of $A$.

It is unknown whether $\operatorname{Tdeg}(A)=\operatorname{Tdeg}\left(S^{-1} A\right)$ holds for every domain $A$ and every Ore set $S$ of $A$. For convenience, we introduce the following technical notion.

Definition 3.4. An algebra $A$ is called Tdeg-stable if the following two conditions hold:

(1) $\operatorname{Tdeg}(A)=\operatorname{GKdim}(A)$, and

(2) for every Ore set $S$ of regular elements, $\operatorname{Tdeg}\left(S^{-1} A\right)=\operatorname{Tdeg}(A)$.

Note that $A$ is Tdeg-stable if and only if $\operatorname{GKdim}(A) \leq \operatorname{Tdeg}\left(S^{-1} A\right)$ for every regular localization $S^{-1} A$. Suppose $Q$ is a finitely generated division algebra with finite Tdeg. By Theorem 3.3(1), Tdeg $(Q)=\inf \operatorname{GKdim}(B)$, where $B$ ranges over subalgebras $B$ of $Q$ with $Q(B)=Q$. If $B$ takes the infimum among these subalgebras, namely $\operatorname{GKdim}(B)=\operatorname{Tdeg}(Q)$, then $B$ is Tdeg-stable. We list below a few easy facts.

Proposition 3.5. Let $A$ be an algebra and suppose that the total quotient algebra $Q(A)$ of $A$ exists.

(1) If $\operatorname{Tdeg}(A)=\operatorname{Tdeg}(Q(A))$ then, for every algebra $B$ with $A \subset B \subset Q(A)$, $\operatorname{Tdeg}(B)=\operatorname{Tdeg}(A)$.

(2) $A$ is Tdeg-stable if and only if $\operatorname{GKdim}(A) \leq \operatorname{Tdeg}(Q(A))$.

(3) If $A$ is Tdeg-stable and $B$ is a subalgebra of $Q(A)$ such that $Q(A)=Q(B)$, then $\operatorname{GKdim}(B) \geq \operatorname{GKdim}(A)$. If moreover $\operatorname{GKdim}(B)=\operatorname{GKdim}(A)$, then $B$ is Tdeg-stable.

(4) If $A$ is Tdeg-stable and $B$ is a subalgebra of $A$ such that $Q(A)=Q(B)$, then $\operatorname{GKdim}(B)=\operatorname{GKdim}(A)$ and $B$ is Tdeg-stable.

Proof. The proof of these statements are routine, so we only give the proof of (2) as an example.

(2) If $A$ is Tdeg-stable, by definition $\operatorname{GKdim}(A)=\operatorname{Tdeg}(A)=\operatorname{Tdeg}(Q(A))$. Conversely, we suppose $\operatorname{GKdim}(A) \leq \operatorname{Tdeg}(Q(A))$. By Propositions 2.1 and 3.1(3),

$$
\operatorname{Tdeg}(Q(A)) \leq \operatorname{Tdeg}\left(S^{-1} A\right) \leq \mathrm{Tdeg}(A) \leq \operatorname{GKdim}(A) \leq \operatorname{Tdeg}(Q(A))
$$

for every localization $S^{-1} A$. Hence $\operatorname{Tdeg}\left(S^{-1} A\right)=\operatorname{Tdeg}(A)=\operatorname{GKdim}(A)$ and thus $A$ is Tdeg-stable.

If $A$ is commutative, by Proposition 2.2 we have $\operatorname{Tdeg}(A)=\operatorname{GKdim}(A)$ and by [NV2, 4.2] we obtain $\operatorname{Tdeg}\left(S^{-1} A\right)=\operatorname{Tdeg}(A)$. Hence $A$ is Tdeg-stable. The Weyl algebras are also Tdeg-stable (as shown in the introduction). We will see many other Tdeg-stable algebras in later sections. However, not every domain of finite GKdim is Tdeg-stable. For example the algebra $S^{-1} A_{1}$ in Example 2.3(2) is not Tdeg-stable. Since $A_{1} \subset S^{-1} A_{1} \subset D_{1}$, by Proposition 3.5(1), $\operatorname{Tdeg}\left(S^{-1} A_{1}\right)=\operatorname{Tdeg}\left(A_{1}\right)=2$. $\operatorname{But} \operatorname{GKdim}\left(S^{-1} A_{1}\right)=3$ (see Example 2.3(2)). Therefore $S^{-1} A_{1}$ is not Tdegstable. 


\section{LOW TDEGS}

In this section we will prove that $\operatorname{Tdeg}(A)=0$ if and only if $\operatorname{GKdim}(A)=0$, and that $\operatorname{Tdeg}(A)=1$ if and only if $\operatorname{Tdeg}(A)=1$ for semiprime Goldie algebras $A$. As a consequence, every semiprime Goldie algebra of GKdim at most 2 is Tdeg-stable.

Theorem 4.1. Let $A$ be an algebra. Then $\operatorname{Tdeg}(A)=0$ if and only if $\operatorname{GKdim}(A)=$ 0 .

Proof. If $\operatorname{GKdim}(A)=0$, by Proposition 2.1, $\operatorname{Tdeg}(A)=0$. Suppose now that $\operatorname{Tdeg}(A)=0$. For every subframe $V$ of $A, I_{V}(A) \leq \operatorname{Tdeg}(A)=0$. Hence there is a regular element $b$ such that $\operatorname{GKdim}(k[b V])=0$. Since $k[b V] \supset k[b], \operatorname{dim}(k[b])$ is finite. Hence $b$ is invertible, and $b^{-1} \in k[b]$ because $b$ is regular. Thus $k[V] \subset k[b V]$ has GKdim 0. Since $V$ is arbitrary, $\operatorname{GKdim}(A)=0$.

One immediate consequence of Theorem 4.1 is the following.

Corollary 4.2. Let $A$ be an algebra.

(1) If $\operatorname{GKdim}(A)=0$ (or equivalently $\operatorname{Tdeg}(A)=0$ ), then $A$ is Tdeg-stable.

(2) If $\operatorname{GKdim}(A)=1$, then $\operatorname{Tdeg}(A)=1$.

(3) If $\operatorname{Tdeg}(A)=1$, then for every localization $S^{-1} A, \operatorname{Tdeg}\left(S^{-1} A\right)=1$.

(4) If $\operatorname{GKdim}(A)=1$, then $A$ is Tdeg-stable.

Proof. (1) Obvious from Theorem 4.1 and Proposition 3.1(3).

(2) By Theorem 4.1, $\operatorname{Tdeg}(A) \neq 0$; and then by Proposition $2.4, \operatorname{Tdeg}(A) \geq 1$.

By Proposition 2.1, $\operatorname{Tdeg}(A) \leq \operatorname{GKdim}(A)=1$ and therefore $\operatorname{Tdeg}(A)=1$.

(3) By Proposition 3.1(3), $\operatorname{Tdeg}\left(S^{-1} A\right) \leq 1$. By Proposition 2.1,

$$
\operatorname{GKdim}\left(S^{-1} A\right) \geq \operatorname{GKdim}(A) \geq \operatorname{Tdeg}(A)=1 .
$$

By Theorem 4.1, $\operatorname{Tdeg}\left(S^{-1} A\right) \geq 1$ and hence $\operatorname{Tdeg}\left(S^{-1} A\right)=1$.

(4) A consequence of (2) and (3).

Theorem 4.3. If $A$ is semiprime Goldie, then $\operatorname{Tdeg}(A)=1$ if and only if $\operatorname{GKdim}(A)=1$.

Proof. One direction is Corollary 4.2(2). Now we suppose $\operatorname{Tdeg}(A)=1$ and $A$ is semiprime Goldie. We need to show $\operatorname{GKdim}(A)=1$. Since $\operatorname{GKdim}(A) \geq$ $\operatorname{Tdeg}(A)=1$, it suffices to prove $\operatorname{GKdim}(A) \leq 1$. Let $Q$ be the semisimple artinian quotient algebra of $A$. By Corollary 4.2(3), $\operatorname{Tdeg}(Q)=1$. So it is enough to show $\operatorname{GKdim}(Q) \leq 1$. By Goldie's Theorem [MR, 2.3.6], $Q \cong \bigoplus_{i=1}^{m} M_{n_{i}}\left(D_{i}\right)$ where the $D_{i}$ are some division algebras. Applying Propositions 2.6 and 2.7(2), we may assume $A$ is a division algebra without loss of generality. Hence we must verify that a division algebra $A$ has GKdim $\leq 1$ if it has Tdeg $\leq 1$.

By definition, $\operatorname{Tdeg}(A)=\sup _{V} \inf _{b} \operatorname{GKdim}(k[b V])$. Hence for every subframe $V$ of $A$, we have $\inf _{b} \operatorname{GKdim}(k[b V]) \leq 1$. Therefore $\operatorname{GKdim}(k[b V]) \leq 1$ for some regular element $b$. Since $k[b V](\subset A)$ is a domain, by [SSW], $k[b V]$ is a noetherian PI domain and a finite module over its center. Let $B$ be the quotient algebra of $k[b V]$. Then $B$ is a central localization of $k[b V][M R, 13.6 .5]$ and hence $\operatorname{GKdim}(B)=$ $\operatorname{GKdim}(k[b V]) \leq 1[\mathrm{MR}, 8.2 .13]$. We have a chain of subalgebras $k[b V] \subset B \subset A$ (we can view $B$ is a subalgebra of $A$ because $A$ is a division algebra). Now $b \in$ $k[b V]$ implies $V=b^{-1} b V \subset B$ and hence $k[V] \subset B$. Therefore $\operatorname{GKdim}(k[V]) \leq$ $\operatorname{GKdim}(B) \leq 1$. Since $V$ is arbitrary, $\operatorname{GKdim}(A) \leq 1$. 
Example 2.10 shows that $\operatorname{Tdeg}\left(D_{1}\right)=2$ and $\operatorname{GKdim}\left(D_{1}\right)=\infty$. So we cannot expect a theorem similar to Theorem 4.3 to hold for Tdeg 2. As an immediate consequence of Theorem 4.3, we have the following.

Corollary 4.4. Let $A$ be a semiprime Goldie algebra.

(1) If $\operatorname{GKdim}(A)=2$, then $\operatorname{Tdeg}(A)=2$.

(2) If $\operatorname{Tdeg}(A)=2$, then for every localization $S^{-1} A$ of $A$, $\operatorname{Tdeg}\left(S^{-1} A\right)=2$.

(3) If $\operatorname{GKdim}(A)=2$, then $A$ is Tdeg-stable.

Proof. Note that if $A$ is semiprime Goldie then every localization of $A$ is semiprime Goldie. The rest of the proof is similar to the proof of Corollary 4.2.

As a consequence of Corollary 4.4(3), if $A$ is a domain of GKdim 2 then $A$ is Tdeg-stable. For example, the skew polynomial algebra $k_{q}[x, y]$ is a domain of GKdim 2 and hence it is Tdeg-stable. However, noetherian domains of GKdim 3 need not be Tdeg-stable [Example 2.3(2)].

\section{PI ANd Locally PI Algebras}

In this section we will prove that every PI (polynomial identity) or locally PI domain (the definition of a locally PI algebra will be given after Theorem 5.3) is Tdeg-stable. At the end of the section we will give an example of a locally PI algebra.

Lemma 5.1. Let $A$ be an algebra and let $Z$ be a subalgebra contained in the center of $A$. Then $\operatorname{Tdeg}(A) \geq \operatorname{GKdim}(Z)$.

Proof. For each finitely generated subalgebra $B$ of $Z$, by the Noether normalization theorem, there is a subframe $V$ of $B$ such that $\operatorname{dim}\left(V^{n}\right) \geq c n^{d}$ for some constant $c>0$ where $d=\operatorname{GKdim}(B)$. For every regular element $b$ in $A$, we have

$$
\operatorname{dim}(k+b V)^{n} \geq \operatorname{dim}(b V)^{n}=\operatorname{dim} b^{n} V^{n}=\operatorname{dim} V^{n} \geq c n^{d} .
$$

Hence $\operatorname{GKdim}(k[b V]) \geq d$ and $\operatorname{Tdeg}(A) \geq I_{V}(A) \geq d$. Therefore $\operatorname{Tdeg}(A) \geq$ $\operatorname{GKdim}(Z)$.

Corollary 5.2. If an algebra $A$ is a finite module over its center, then $A$ is Tdegstable.

Proof. Let $Z$ be the center of $A$. For every localization $S^{-1} A, Z$ is contained in the center of $S^{-1} A$. Since $A$ is a finite module over $Z, \operatorname{GKdim}(A)=\operatorname{GKdim}(Z)$ [MR, 8.2 .9 (ii)]. Hence, by Lemma 5.1, we have

$$
\operatorname{GKdim}(A)=\operatorname{GKdim}(Z) \leq \operatorname{Tdeg}\left(S^{-1} A\right) .
$$

By the remark after Definition 3.4, $A$ is Tdeg-stable.

Now we are ready to show that semiprime Goldie PI algebras are Tdeg-stable. Note that a prime PI algebra is automatically Goldie [MR, 13.6.6(i)].

Theorem 5.3. Every semiprime Goldie PI algebra is Tdeg-stable.

Proof. Let $A$ be a semiprime Goldie PI algebra and let $Q$ be the quotient algebra of $A$. By a result of W. S. Martindale [Ma, 2], $Q$ is a PI algebra. Since $Q$ is semisimple 
artinian, namely, a finite direct sum of simple algebras, by Kaplansky's theorem (see [MR, 13.3.8]) $Q$ is a finite module over its center. Hence by Corollary 5.2, $Q$ is Tdeg-stable, whence

$$
\operatorname{GKdim}(A) \leq \operatorname{GKdim}(Q)=\operatorname{Tdeg}(Q) .
$$

By Proposition 3.5(2), $A$ is Tdeg-stable.

In the rest of this section, we consider a class of algebras which are very close to being PI algebras. An algebra $A$ is said to be a locally $P I$ algebra if every finitely generated subalgebra of $A$ is a PI algebra. Hence a PI algebra is a locally PI algebra and an affine locally PI algebra is a PI algebra. Every subalgebra of a locally PI algebra is again a locally PI algebra. By [SSW], algebras with GKdim $\leq 1$ are locally PI.

Let $I$ be a directed set, i.e., for every pair of elements $i, j$ in $I$, there is an $l$ in $I$ such that $l \geq i, j$. Suppose $\left\{A_{i} \mid i \in I\right\}$ is a set of subalgebras of $A$ such that $A_{i} \subset A_{j}$ if and only if $i \leq j$ in $I$. An algebra $A$ is said to be the union of subalgebras $\left\{A_{i} \mid i \in I\right\}$ if $A=\bigcup_{i \in I} A_{i}$. Since $A$ is a union of finitely generated subalgebras, a locally PI algebra is a union of PI subalgebras.

Lemma 5.4. Let $I$ be a directed set and suppose $A$ is the union of subalgebras $\left\{A_{i} \mid i \in I\right\}$.

(1) $\operatorname{GKdim}(A)=\max _{i \in I}\left\{\operatorname{GKdim}\left(A_{i}\right)\right\}$.

(2) If each $A_{i}$ is an Ore domain, then so is $A$, and $Q(A)=\bigcup_{i \in I} Q\left(A_{i}\right)$.

(3) Let $Z(A)$ and $Z\left(A_{i}\right)$ be the centers of $A$ and $A_{i}$. Then

$$
Z(A)=\lim _{\longrightarrow}\left[\bigcap_{j \geq i} Z\left(A_{j}\right)\right] .
$$

Proof. (1) and (2) are routine.

(3) It is obvious that $Z(A) \supset \bigcap_{j \geq i} Z\left(A_{j}\right)$ for all $i$. Now suppose $x \in Z(A)$. There is an $i$ such that $x \in A_{i}$. Since $A \supset A_{j}$ for all $j, x \in Z\left(A_{j}\right)$ for all $j \geq i$. Thus $Z(A)=\lim _{\longrightarrow}\left[\bigcap_{j \geq i} Z\left(A_{j}\right)\right]$.

Proposition 5.5. Let $I$ be a directed set and suppose that $A$ is the union of subalgebras $\left\{A_{i} \mid i \in I\right\}$.

(1) If $A$ is a domain, then $\operatorname{Tdeg}(A) \leq \max _{i \in I}\left\{\operatorname{Tdeg}\left(A_{i}\right)\right\}$. Equality holds when the right-hand side is 0,1 or 2 .

(2) If $A$ is a Tdeg-stable domain, then $\operatorname{Tdeg}(A)=\max _{i \in I}\left\{\operatorname{Tdeg}\left(A_{i}\right)\right\}$.

Note that there is a non-PI division algebra $A$ such that $2=\operatorname{Tdeg}(A)<$ $\max \left\{\operatorname{Tdeg}\left(A_{i}\right)\right\}$. One can easily construct an example based on the algebras in Example 2.10.

Proof. (1) Let $V$ be a subframe of $A$. Then there is an $i$ such that $V \subset A_{i}$. Hence $I_{V}(A) \leq I_{V}\left(A_{i}\right)$, because $A_{i} \subset A$. Therefore $I_{V}(A) \leq \operatorname{Tdeg}\left(A_{i}\right) \leq \max \left\{\operatorname{Tdeg}\left(A_{i}\right)\right\}$, and this implies that $\operatorname{Tdeg}(A) \leq \max \left\{\operatorname{Tdeg}\left(A_{i}\right)\right\}$.

If $\max \left\{\operatorname{Tdeg}\left(A_{i}\right)\right\}$ is 0 or 1 , by Theorems 4.1 and $4.3, \operatorname{Tdeg}\left(A_{i}\right)=\operatorname{GKdim}\left(A_{i}\right) \leq$ 1 for all $i$ and hence $\operatorname{Tdeg}(A)=\operatorname{GKdim}(A) \leq 1$. By Lemma 5.4(1), $\operatorname{Tdeg}(A)=$ 
$\max \left\{\operatorname{Tdeg}\left(A_{i}\right)\right\}$. If $\max \left\{\operatorname{Tdeg}\left(A_{i}\right)\right\}=2, \operatorname{Tdeg}(A) \leq 2$. But by Theorem 4.3, $\operatorname{Tdeg}(A)>1$ and by Proposition 2.4, $\operatorname{Tdeg}(A)=2$.

(2) If $A$ is a Tdeg-stable domain, then

$\operatorname{Tdeg}(A) \leq \max \left\{\operatorname{Tdeg}\left(A_{i}\right)\right\} \leq \max \left\{\operatorname{GKdim}\left(A_{i}\right)\right\}=\operatorname{GKdim}(A)=\operatorname{Tdeg}(A)$.

Therefore (2) follows.

Note that a prime locally PI algebra may not be Goldie. An easy example can be obtained by constructing $\lim _{n \rightarrow \infty} M_{n}(k)$ in a proper way.

Now we are ready to prove a version of Theorem 5.3 for locally PI algebras. For simplicity, we only consider locally PI domains.

Theorem 5.6. Every locally PI domain $A$ is Ore and Tdeg-stable, and the quotient division algebra $Q(A)$ is locally $P I$ with $\operatorname{GKdim}(Q(A))=\operatorname{GKdim}(A)$.

Proof. A locally PI algebra $A$ is a union of PI subalgebras $\left\{A_{i}\right\}$. Since every PI domain $A_{i}$ is Ore [MR, 13.6.6], by Lemma 5.4(2), $A$ is an Ore domain and $Q(A)$ is the union of $\left\{Q\left(A_{i}\right)\right\}$. Consequently $Q(A)$ is a locally PI algebra. Since the PI algebras $A_{i}$ and $Q\left(A_{i}\right)$ are Tdeg-stable [Theorem 5.3],

$$
\operatorname{GKdim}\left(Q\left(A_{i}\right)\right)=\operatorname{Tdeg}\left(Q\left(A_{i}\right)\right)=\operatorname{GKdim}\left(A_{i}\right) .
$$

By Lemma $5.4(1), \operatorname{GKdim}(Q(A))=\operatorname{GKdim}(A)$. It remains to prove that $A$ is Tdeg-stable. Let $V$ be a subframe of $A$ and let $b$ be a nonzero element of $A$. Since $k[b V]$ is finitely generated, $k[b V]$ is a PI domain. By [MR, 13.6.6], $k[b V]$ is an Ore domain and

$$
\operatorname{GKdim}(k[b V])=\operatorname{GKdim}(Q(k[b V])) \geq \operatorname{GKdim}(k[V])
$$

because $Q(k[b V]) \supset k[V]$. Therefore

$$
\operatorname{Tdeg}(A)=\sup _{V} \inf _{b} \operatorname{GKdim}(k[b V])=\sup _{V} \operatorname{GKdim}(k[V])=\operatorname{GKdim}(A) .
$$

Applying the above to $Q(A)$ we obtain $\operatorname{Tdeg}(Q(A))=\operatorname{GKdim}(Q(A))$. Hence $\operatorname{Tdeg}(Q(A))=\operatorname{GKdim}(A)$, and by Proposition 3.5(2), $A$ is Tdeg-stable.

Finally we give an example of a locally PI (but not PI) division algebra by constructing a chain of subalgebras. For convenience, in the following example we assume that the base field $k$ is algebraically closed and $\operatorname{char}(k)=0$.

Example 5.7. There is a locally PI division algebra $Q$ such that

(1) $\operatorname{Tdeg}(Q)=\operatorname{GKdim}(Q)=2$,

(2) the center of $Q$ is the base field $k$, and

(3) $Q$ is not a PI algebra.

Let $r_{n}$ be a primitive $4^{n}$-th root of 1 such that $r_{n}^{4}=r_{n-1}$ for all $n>0$. Let $C_{n}=$ $k_{r_{n}}\left[x_{n}, y_{n}\right]$ with the relation $y_{n} x_{n}=r_{n} x_{n} y_{n}$ for all $n \geq 0$. Then $y_{n}^{2} x_{n}^{2}=r_{n-1} x_{n}^{2} y_{n}^{2}$ for all $n$. Hence we can identify $x_{n-1}$ with $x_{n}^{2}$ and $y_{n-1}$ with $y_{n}^{2}$ for all $n$ such that

$$
C_{0} \subset C_{1} \subset \cdots C_{n} \subset C_{n+1} \cdots .
$$

Let $Q_{n}$ be the quotient algebra of $C_{n}$. Then $Q=\lim _{n \rightarrow \infty} Q_{n}$ satisfies the properties listed above. First, $Q$ is a locally PI algebra because $C_{n}$ and $Q_{n}$ are PI algebras. To see (1) we note that $\operatorname{GKdim}(Q)=2$, and by Corollary $4.4(3) \operatorname{Tdeg}(Q)=2$. (2) can be proved by using Lemma 5.4(3), and (3) is a consequence of (2). 


\section{Filtered Algebras}

In this section we will generalize the method used by Gelfand and Kirillov in their original paper [GK]. As an application we will re-prove their theorem and the Borho-Kraft theorem [Corollary 6.10].

Let us start by reviewing the definition of a filtered algebra. Let $G$ be an ordered semigroup and let $A$ be an algebra with a filtration $\left\{F_{g} \mid g \in G\right\}$ of subspaces of $A$. Suppose the filtration satisfies the following conditions:

(f1) $F_{g} \subset F_{h}$ for all $g<h$ in $G$;

(f2) $F_{g} F_{h} \subset F_{g h}$ for all $g, h \in G$;

for every $g \in G$, we define $F_{<g}$ to be $\sum_{h<g} F_{h}$,

(f3) $A=\bigcup_{g \in G}\left(F_{g}-F_{<g}\right)$;

(f4) $1 \in F_{e}-F_{<e}$, where $e$ is the unit of $G$.

Then we can define an associated graded algebra $\operatorname{gr}(A):=\bigoplus_{g \in G} F_{g} / F_{<g}$ with the $k$-linear multiplication determined by $\left(a+F_{<g}\right)\left(b+F_{<h}\right)=a b+F_{<g h}$ (see [KL, page 73] for the case $G=\mathbb{Z}$ ). For our purposes we also assume

(f5) $\operatorname{gr}(A)$ is a $G$-graded domain.

We define a map $\nu: A \longrightarrow \operatorname{gr}(A)$ by $\nu(a)=a+F_{<g}$ for all $a \in F_{g}-F_{<g}$. This map is called a leading-term map, and it is easy to see that

(11) $\nu(t)=t$ for all $t \in k$;

(12) $\nu(a) \neq 0$ for all $a \neq 0$.

By the definition of $\operatorname{gr}(A)$ and (f5), we see that

(13) $\nu(a b)=\nu(a) \nu(b)$ for all $a, b \in A$.

For every subspace $V$ of $A$, we define $\nu(V)=\sum_{x \in V} k \nu(x)$. Hence $\nu(V)$ is a graded subspace of $g r(A)$. For every $g \in G$, let $V_{\leq g}=V \cap F_{g}$ and $V_{<g}=V \cap F_{<g}$. Then $V_{\leq g}$ and $V_{<g}$ are subspaces of $V$, and

$$
V_{\leq g} \subset V_{<h} \subset V_{\leq h}
$$

for all $g<h$. By the definition of $\nu$ we see that $\nu(V)=\bigoplus_{g} V_{\leq g} / V_{<g}$. Hence $\operatorname{dim}(\nu(V))=\sum_{g}\left(\operatorname{dim}\left(V_{\leq g}\right)-\operatorname{dim}\left(V_{<g}\right)\right)=\operatorname{dim} V$. Therefore the leading-term map $\nu$ satisfies

(14) $\operatorname{dim}(\nu(V))=\operatorname{dim}(V)$ for every subspace $V$ of $A$.

Note that $(11,2,4)$ will still hold, but (13) will fail, if (f5) fails.

Next we give some examples of filtered algebras which will be used later.

Example 6.1. $G$-graded domains.

Let $G$ be an ordered semigroup. A $G$-graded domain $A$ can be viewed naturally as a $G$-filtered algebra. For every $g \in G$, let $F_{g}$ be $\bigoplus_{h<g} A_{h}$. Then $\left\{F_{g} \mid g \in G\right\}$ is a filtration and $\operatorname{gr}(A)=A$. Since $A$ is a domain, (f5) holds. The leading-term map is $\nu(a)=a_{g_{n}}$ for $a=a_{g_{1}}+a_{g_{2}}+\cdots+a_{g_{n}}$ with $0 \neq a_{g_{i}} \in A_{g_{i}}$ and $g_{1}<g_{2}<\cdots<g_{n} . \square$

Example 6.2. $\mathbb{N}$-filtered algebras.

Let $A$ be a domain generated by a subframe $V$ and let $F_{0}=k$ and $F_{i}=V^{i}$ for all $i \geq 1$. Then $\left\{F_{i} \mid i \geq 0\right\}$ is a filtration of $A$. (We may add $F_{n}=0$ for $n<0$ to make this a $\mathbb{Z}$-filtration.) Suppose that the associated graded algebra $\operatorname{gr}(A):=\bigoplus_{i} V^{i} / V^{i-1}$ is a domain. Then (f1,2,3,4,5) hold, and $\nu: a \longmapsto a+V^{i-1}$ for all $a \in V^{i}-V^{i-1}$ is the leading-term map from $A$ to $\operatorname{gr}(A)$. Familiar examples are the Weyl algebras and universal enveloping algebras of finite dimensional Lie algebras. 
Example 6.3. Descending chains of ideals.

Let $\left\{P_{i} \mid i \geq 0\right\}$ be a descending chain of ideals of $A$ such that $P_{i} P_{j} \subset P_{i+j}$ and $\bigcap_{i} P_{i}=\{0\}$. Then $F_{-n}=P_{n}$ for $n>0$ and $F_{n}=A$ for all $n \geq 0$ defines a filtration of $A$. The associated graded algebra is $\bigoplus_{i} P_{i} / P_{i+1}$ with natural multiplication. If $\operatorname{gr}(A)=\bigoplus_{i} P_{i} / P_{i+1}$ is a domain, then the filtration satisfies (f1,2,3,4,5). For example, let $x$ be a normal regular element $x$ of $A$ such that $A /(x)$ is a domain and $\bigcap_{i} x^{i} A=\{0\}$. It is easy to check that $\operatorname{gr}(A):=\bigoplus_{i \geq 0} x^{i} A / x^{i+1} A$ is isomorphic to $A /(x)[z, \sigma]$ where $\sigma$ is the automorphism induced by the automorphism $\beta$ of $A$ determined by $a x=x a^{\beta}$. Hence $\left\{P_{i}:=x^{i} A \mid i \geq 0\right\}$ is a descending chain of ideals satisfies the required conditions.

For our purpose of computing Tdeg for various algebras, we generalize the leading-term map to the following version which will be used in cases other than filtered algebras. Let $A$ and $B$ be algebras and let $\nu$ be a map from $A$ to $B$ (which may not be a $k$-linear map). We call $\nu$ a valuation from $A$ to $B$ if the following conditions hold

(v1) $\nu(t)=t$ for all $t \in k$;

(v2) $\nu(a) \neq 0$ for all nonzero $a \in A$;

(v3) for any $a, b \in A, \nu(a b)=t \nu(a) \nu(b)$ for some nonzero scalar $t \in k$;

(v4) for every subspace $V$ of $A$,

$$
\operatorname{dim}(\nu(V))=\operatorname{dim}(V),
$$

where $\nu(V):=\sum_{x \in V} k \nu(x)$.

In fact we only need the following weak version (v4)' to prove all statements about valuation in this paper.

(v4)' For every subspace $V$ of $A, \operatorname{dim}(\nu(V)) \leq \operatorname{dim}(V)$.

Comparing $(11,2,3,4)$ and $(\mathrm{v} 1,2,3,4)$, we see that leading-term maps of filtered algebras are valuations. Other examples of valuations will be given in next two sections.

Lemma 6.4. Let $A$ and $B$ be algebras and let $\nu$ be a valuation from $A$ to $B$.

(1) $\nu(c V)=\nu(c) \nu(V)$ for all $c \in A$ and $V \subset A$.

(2) $\nu(V+W) \supset \nu(V)+\nu(W)$ for all $V, W \subset A$.

(3) $\nu(V W) \supset \nu(V) \nu(W)$ for all $V, W \subset A$.

(4) $\operatorname{dim}\left(V^{n}\right) \geq \operatorname{dim}\left((\nu(V))^{n}\right)$ for all $V \subset A$ and all $n \geq 1$.

Proof. (1) $\nu(c V)=\sum_{v} k \nu(c v)=\sum_{v} k \nu(c) \nu(v)=\nu(c) \sum_{v} k \nu(v)=\nu(c) \nu(V)$.

(2) Since $V \subset V+W$, it follows that $\nu(V) \subset \nu(V+W)$, and then $\nu(V)+\nu(W) \subset$ $\nu(V+W)$.

(3) Write $V W=\sum_{v} v W$; then (3) follows from (1) and (2).

(4) By (v4)', $\operatorname{dim}\left(V^{n}\right) \geq \operatorname{dim}\left(\nu\left(V^{n}\right)\right)$ and by $(3), \operatorname{dim}\left(\nu\left(V^{n}\right)\right) \geq \operatorname{dim}\left((\nu(V))^{n}\right)$. Hence (4) follows from these inequalities.

Proposition 6.5. Let $A$ and $B$ be algebras and let $\nu$ be a valuation from $A$ to $B$. Suppose that $S$ is an Ore set of regular elements of $A$ and that $\nu(S):=\{\nu(x) \mid x \in S\}$ is an Ore set of regular elements of $B$. Then $\nu$ can be extended to a valuation from $S^{-1} A$ to $(\nu(S))^{-1} B$.

Proof. First we define a map $\mu$ from $S^{-1} A$ to $\nu(S)^{-1} B$ as follows. For every element $x \in S^{-1} A$, pick a pair of elements $(s, a)$ such that $x=s^{-1} a$ and define

$$
\mu\left(s^{-1} a\right)=\nu(s)^{-1} \nu(a) .
$$


It is routine to check that if $s^{-1} a=t^{-1} b$ then $\nu(s)^{-1} \nu(a)$ and $\nu(t)^{-1} \nu(b)$ differ by a nonzero scalar in $k$. By choosing $(s, a)$ carefully we may define $\mu(a)=\nu(a)$ for all $a \in A$. Now (v1) and (v2) hold obviously. It remains to check (v3) and (v4). Suppose $x=a^{-1} b$ and $y=c^{-1} d$ in $S^{-1} A$. Since $S$ is Ore, there are $f \in S$ and $g \in A$ such that $b c^{-1}=f^{-1} g$. Hence $f b=g c$ and $k \nu(f) \nu(b)=k \nu(g) \nu(c)$ as a 1-dimensional subspace. Then in $B$ we have $k \nu(b) \nu(c)^{-1}=k \nu(f)^{-1} \nu(g)$. Therefore

$$
\begin{aligned}
k \mu(x y) & =k \mu\left(a^{-1} b c^{-1} d\right)=k \mu\left(a^{-1} f^{-1} g d\right) \\
& =k \nu(a)^{-1} \nu(f)^{-1} \nu(g) \nu(d)=k \nu(a)^{-1} \nu(b) \nu(c)^{-1} \nu(d) \\
& =k \mu(x) \mu(y) .
\end{aligned}
$$

Thus (v3) holds. Let $V$ be a finite dimensional subspace of $S^{-1} A$. Then there is an $s \in S$ such that $V=s^{-1} V_{1}$ and $V_{1} \subset A$. Hence

$$
\begin{aligned}
\operatorname{dim}(\mu(V)) & =\operatorname{dim}\left(\mu\left(s^{-1} V_{1}\right)\right)=\operatorname{dim}\left(\mu\left(s^{-1}\right) \mu\left(V_{1}\right)\right) \\
& =\operatorname{dim}\left(\nu(s)^{-1} \nu\left(V_{1}\right)\right)=\operatorname{dim}\left(\nu\left(V_{1}\right)\right) \\
& =\operatorname{dim}\left(V_{1}\right)=\operatorname{dim}(V) .
\end{aligned}
$$

Therefore (v4) holds and $\mu$ is a valuation.

Corollary 6.6. Let $A$ and $B$ be domains and let $\nu$ be a valuation from $A$ to $B$. Suppose that $S$ is an Ore set of $A$ and that $\nu(x)$ is invertible in $B$ for all $x \in S$. Then $\nu$ can be extented to a valuation from $S^{-1} A$ to $B$.

Let $\nu$ be a valuation from $A$ to $B$ and suppose $\nu(A)=B$. Then for every subframe $W$ of $B$, there is a subframe $V$ of $A$ such that $\nu(V) \supset W$. Thus we have

$$
\operatorname{GKdim}(B)=\sup _{V \subset A} \operatorname{GKdim}(k[\nu(V)])
$$

and

$$
\operatorname{Tdeg}(B)=\sup _{V \subset A} \inf _{b} \operatorname{GKdim}(k[b \nu(V)]) .
$$

Theorem 6.7(1), below, is an analogue of [MR, 8.3.20].

Theorem 6.7. Let $A$ and $B$ be domains and suppose $\nu$ is a valuation from $A$ to $B$ such that $\nu(A)=B$. Then

(1) $\operatorname{GKdim}(A) \geq \operatorname{GKdim}(B)$.

(2) $\operatorname{Tdeg}(A) \geq \operatorname{Tdeg}(B)$.

(3) If $\nu(x)$ is invertible in $B$ for all $x \in A-0$, then $\operatorname{Tdeg}(Q(A)) \geq \operatorname{Tdeg}(B)$.

Proof. (1) By definition, $\operatorname{GKdim}(A)=\sup _{V \subset A} \operatorname{GKdim}(k[V])$. By Lemma 6.4(4), we have $\operatorname{dim}\left(V^{n}\right) \geq \operatorname{dim}\left(\nu(V)^{n}\right)$. By (6.6.1), we obtain $\operatorname{GKdim}(A) \geq \operatorname{GKdim}(B)$.

(2) By definition, $\operatorname{Tdeg}(A)=\sup _{V \subset A} \inf _{a} \operatorname{GKdim}(k[a V])$. By Lemma 6.4,

$$
\operatorname{dim}\left((k+a V)^{n}\right) \geq \operatorname{dim}\left((\nu(k+a V))^{n}\right) \geq \operatorname{dim}\left((k+\nu(a) \nu(V))^{n}\right) .
$$

Hence $\operatorname{GKdim}(k[a V]) \geq \operatorname{GKdim}(k[\nu(a) \nu(V)]) \geq I_{\nu(V)}(B)$. Therefore $I_{V}(A) \geq$ $I_{\nu(V)}(B)$ for all subframes $V \subset A$. By (6.6.2), we have $\operatorname{Tdeg}(A) \geq \operatorname{Tdeg}(B)$.

(3) By Corollary 6.6, there is a valuation from $Q(A)$ to $B$. Replacing $A$ by $Q(A)$ in (2), we obtain (3). 
Theorem 6.8. Let $A$ and $B$ be domains and let $\nu: A \longrightarrow B$ be a valuation such that $\nu(A)=B$. If $\operatorname{GKdim}(A)=\operatorname{GKdim}(B)<\infty$ and $B$ is Tdeg-stable, then $A$ is Tdeg-stable.

Proof. Let $Q(A)$ be the quotient algebra of $A$ and $Q(B)$ the quotient algebra of $B$. Since $\nu$ is a valuation from $A$ to $B, \nu$ is also a valuation from $A$ to $Q(B)$. By Corollary 6.6, an extension of $\nu$, still denoted by $\nu$, is a valuation from $Q(A)$ to $Q(B)$. Let $C$ denote the vector space $\nu(Q(A))$. Since every element in $C$ is of the form $\sum a_{i} \nu\left(x_{i}\right), C$ is an algebra between $B$ and $Q(B)$. By Theorem 6.7(2), $\operatorname{Tdeg}(Q(A)) \geq \operatorname{Tdeg}(C)$ and by Proposition 3.1(3), $\operatorname{Tdeg}(C) \geq \operatorname{Tdeg}(Q(B))$. Since $B$ is Tdeg-stable and $\operatorname{GKdim}(A)=\operatorname{GKdim}(B)$, we have

$$
\operatorname{Tdeg}(Q(A)) \geq \operatorname{Tdeg}(C) \geq \operatorname{Tdeg}(Q(B))=\operatorname{GKdim}(B)=\operatorname{GKdim}(A) .
$$

By Proposition 3.5(2), $A$ is Tdeg-stable.

Now we are ready to prove Gelfand-Kirillov's Theorem [Theorem 2.9] and BorhoKraft's Theorem [BK, 6.5].

Corollary 6.9. The following algebras are Tdeg-stable.

(1) Any algebra A generated by a subframe $V$ such that $\operatorname{gr}(A):=\bigoplus_{i \geq 0} V^{i} / V^{i-1}$ is a Tdeg-stable domain.

(2) Any algebra A generated by a subframe $V$ such that $\operatorname{gr}(A):=\bigoplus_{i \geq 0} V^{i} / V^{i-1}$ is a commutative domain.

(3) (Gelfand-Kirillov) The $n$-th Weyl algebra $A_{n}$.

(4) (Borho-Kraft) The universal enveloping algebra $U(L)$ of a Lie algebra $L$.

Proof. (1) By Example 6.2, there is a valuation $\nu$ from $A$ to $\operatorname{gr}(A)$ and $\nu(A)=$ $\operatorname{gr}(A)$. Since $\operatorname{gr}(A)$ is a domain and $\operatorname{gr}(A)$ is finitely generated (by $\nu(V)), \operatorname{GKdim}(A)$ $=\operatorname{GKdim}(\operatorname{gr}(A))[\mathrm{MR}, 8.6 .5]$. Since $\operatorname{gr}(A)$ is Tdeg-stable, by Theorem $6.8 A$ is Tdeg-stable.

(2) is a special case of (1) because commutative algebras are Tdeg-stable, and (3) and (4) are consequences of (2) by using the standard filtration of $A_{n}$ given in [KL, 7.3] and the standard filtration of $U(L)$ given in [KL, 6.8].

Finally we state the following proposition, which can be proved by combining Theorems 6.7 and 6.8 and Example 6.3.

Proposition 6.10. Let $A$ be an algebra and let $x$ be a normal regular element of A such that (a) $\bigcap_{i} x^{i} A=\{0\}$ and (b) $A /(x)$ is a domain. Suppose that $\sigma$ is the automorphism described in Example 6.3. Then

(1) $\operatorname{GKdim}(A) \geq \operatorname{GKdim}(A /(x)[z, \sigma])$.

(2) $\operatorname{Tdeg}(A) \geq \operatorname{Tdeg}(A /(x)[z, \sigma])$.

(3) If $\operatorname{GKdim}(A)=\operatorname{GKdim}(A /(x)[z, \sigma])$ and $A /(x)[z, \sigma]$ is Tdeg-stable, then $A$ is Tdeg-stable.

\section{Some Quantized Algebras}

In this section we will compute Tdeg of several quantized algebras related to quantum groups. We start with skew polynomial algebras. 
Example 7.1. Skew polynomial algebras and valuations.

Let $\left\{p_{i j} \mid 1 \leq i<j \leq n\right\}$ be a set of nonzero scalars in $k$. The skew polynomial algebra $k_{p_{i j}}\left[x_{1}, \cdots, x_{n}\right]$ is generated by $\left\{x_{1}, \cdots, x_{n}\right\}$ subject to the relations $x_{j} x_{i}=$ $p_{i j} x_{i} x_{j}$ for all $i<j$. The set of ordered monomials $\left\{x_{1}^{l_{1}} \cdots x_{n}^{l_{n}} \mid\left(l_{1}, \cdots, l_{n}\right) \in \mathbb{N}^{n}\right\}$ is a $k$-linear basis of $k_{p_{i j}}\left[x_{1}, \cdots, x_{n}\right]$. Let $I d$ denote the $k$-linear isomorphism defined by sending the ordered monomials of $k_{p_{i j}}\left[x_{1}, \cdots, x_{n}\right]$ to the ordered monomials of the commutative polynomial algebra $k\left[x_{1}, \cdots, x_{n}\right]$ identically. In this sense, $k_{p_{i j}}\left[x_{1}, \cdots, x_{n}\right]$ and $k\left[x_{1}, \cdots, x_{n}\right]$ are the same as a vector space. For every two monomials $x_{1}^{l_{1}} \cdots x_{n}^{l_{n}}$ and $x_{1}^{w_{1}} \cdots x_{n}^{w_{n}}$, we have

$$
\begin{aligned}
\operatorname{Id}\left(x_{1}^{l_{1}} \cdots x_{n}^{l_{n}} \cdot x_{1}^{w_{1}} \cdots x_{n}^{w_{n}}\right) & =I d\left(\prod_{j>i} p_{i j}^{l_{j} w_{i}} x_{1}^{l_{1}+w_{1}} \cdots x_{n}^{l_{n}+w_{n}}\right) \\
& =\prod_{j>i} p_{i j}^{l_{j} w_{i}} I d\left(x_{1}^{l_{1}+w_{1}} \cdots x_{n}^{l_{n}+w_{n}}\right) \\
& =\prod_{j>i} p_{i j}^{l_{j} w_{i}} I d\left(x_{1}^{l_{1}} \cdots x_{n}^{l_{n}}\right) \operatorname{Id}\left(x_{1}^{w_{1}} \cdots x_{n}^{w_{n}}\right) .
\end{aligned}
$$

The algebra $k_{p_{i j}}\left[x_{1}, \cdots x_{n}\right]$ is a $\mathbb{Z}^{n}$-graded domain with $\operatorname{deg}\left(x_{i}\right)=e_{i}$. Recall that $e_{i}=(0, \cdots, 1, \cdots, 0)$ where 1 is in the $i$-th position. Since $\mathbb{Z}^{n}$ is an ordered group $[\mathrm{Pa}, 13.1 .6]$, by Example 6.1, there is a leading-term map $\nu_{p}$ from $k_{p_{i j}}\left[x_{1}, \cdots, x_{n}\right]$ to itself. Since $I d$ is the identity map of the vector space, $I d \circ \nu_{p}$ satisfies (v1,2,4). Note that $\nu_{p}(a)$ is a monomial for every nonzero element $a$. The computation (7.1.1) shows that (v3) holds for $I d \circ \nu_{p}$. Therefore $\mu:=I d \circ \nu_{p}$ is a valuation from $k_{p_{i j}}\left[x_{1}, \cdots, x_{n}\right]$ to $k\left[x_{1}, \cdots, x_{n}\right]$. Conversely, by Example 6.1 , there is a leadingterm map $\nu_{1}$ from $k\left[x_{1}, \cdots, x_{n}\right]$ to itself. A similar argument shows that $\alpha:=$ $I d^{-1} \circ \nu_{1}$ is a valuation from $k\left[x_{1}, \cdots, x_{n}\right]$ to $k_{p_{i j}}\left[x_{1}, \cdots, x_{n}\right]$.

Those two valuations in Example 7.1 allow us to prove the following proposition, which is a special case of Lorenz's result [Lo, 2.2]

Proposition 7.2 (Lorenz). The following algebras are Tdeg-stable.

(1) Skew polynomial algebra $k_{p_{i j}}\left[x_{1}, \cdots, x_{n}\right]$.

(2) Skew Laurent polynomial algebra $k_{p_{i j}}\left[x_{1}, x_{1}^{-1}, \cdots, x_{n}, x_{n}^{-1}\right]$.

Proof. (1) Let $\mu$ and $\alpha$ be the valuations described in Example 7.1. It is easy to check that $\mu\left(k_{p_{i j}}\left[x_{1}, \cdots, x_{n}\right]\right)=k\left[x_{1}, \cdots, x_{n}\right]$ and that $\alpha\left(k\left[x_{1}, \cdots, x_{n}\right]\right)=$ $k_{p_{i j}}\left[x_{1}, \cdots, x_{n}\right]$. By using Theorem 6.7(1) twice, we obtain that

$$
\operatorname{GKdim}\left(k_{p_{i j}}\left[x_{1}, \cdots, x_{n}\right]\right)=\operatorname{GKdim}\left(k\left[x_{1}, \cdots, x_{n}\right]\right) .
$$

Since the commutative algebra $k\left[x_{1}, \cdots, x_{n}\right]$ is Tdeg-stable, by Theorem 6.8 , so is $k_{p_{i j}}\left[x_{1}, \cdots, x_{n}\right]$.

(2) The set of all monomials is an Ore set of both the skew and commutative polynomial algebras. By Proposition $6.5 \mu$ and $\alpha$ can be extended to valuations between the localizations $k_{p_{i j}}\left[x_{1}, x_{1}^{-1}, \cdots, x_{n}, x_{n}^{-1}\right]$ and $k\left[x_{1}, x_{1}^{-1}, \cdots, x_{n}, x_{n}^{-1}\right]$. An argument similar to the proof of (1) shows that $k_{p_{i j}}\left[x_{1}, x_{1}^{-1}, \cdots, x_{n}, x_{n}^{-1}\right]$ is Tdegstable. 
Next we study other deformations of commutative polynomial algebras, a large class of which will be in the following category. We use the lexicographical order on $\mathbb{Z}^{n}$ with $\operatorname{deg}\left(e_{i}\right)<\operatorname{deg}\left(e_{j}\right)$ for $i<j$. An algebra $A$ is called a filtered skew polynomial algebra of $n$ variables if there is a set of generators $\left\{x_{1}, \cdots, x_{n}\right\}$ of $A$ such that the following three conditions hold.

(q1) The set of monomials $\left\{x_{1}^{l_{1}} \cdots x_{n}^{l_{n}} \mid\left(l_{1}, \cdots, l_{n}\right) \in \mathbb{N}^{n}\right\}$ is a $k$-linear basis of $A$. We define $\operatorname{deg}\left(x_{1}^{l_{1}} \cdots x_{n}^{l_{n}}\right)=\left(l_{1}, \cdots, l_{n}\right)$ and

$F_{\left(l_{1}, \cdots, l_{n}\right)}=\left\{\right.$ all linear combinations of the monomials of degree $\left.\leq\left(l_{1}, \cdots, l_{n}\right)\right\}$.

(q2) $\left\{F_{\left(l_{1}, \cdots, l_{n}\right)} \mid\left(l_{1}, \cdots, l_{n}\right) \in \mathbb{N}^{n}\right\}$ is a filtration of $A$.

(q3) The associated graded algebra $\operatorname{gr}(A)$ is isomorphic to a skew polynomial algebra.

It is not difficult to check that the Weyl algebras are filtered skew polynomial algebras.

Theorem 7.3. Let $A$ be a filtered skew polynomial algebra of $n$ variables.

(1) $\operatorname{GKdim}(A) \geq n$.

(2) $\operatorname{Tdeg}(A) \geq n$.

(3) If $\operatorname{GKdim}(A)=n$, then $A$ is Tdeg-stable.

Proof. By definition, $A$ is an $\mathbb{N}^{n}$-filtered algebra with the associated graded algebra being $k_{p_{i j}}\left[x_{1}, \cdots, x_{n}\right]$. By Proposition $7.2(1) k_{p_{i j}}\left[x_{1}, \cdots, x_{n}\right]$ is a Tdeg-stable domain of GKdim $n$. Since the leading-term map is a valuation, (1) and (2) follow from Theorem 6.7 and hence (3) follows from Theorem 6.8.

As a consequence of Theorem 7.3(3), if $A$ and $B$ are two filtered skew polynomial algebras of $n$ and $m$ variables respectively and if $\operatorname{GKdim}(A)=n$ and $\operatorname{GKdim}(B)=$ $m$, then the quotient division algebras of $A$ and $B$ are not isomorphic if $n \neq m$. We will study three examples of filtered skew polynomial algebras which are related to the recent study of quantum groups.

Example 7.4. Quantum matrix algebras $M_{q, p_{i j}}(n)$ and quantum groups $G L_{q, p_{i j}}(n)$ are Tdeg-stable.

For details of $M_{q, p_{i j}}(n)$ and $G L_{q, p_{i j}}(n)$ we refer to the papers [AST] and [Su]. Given a set of nonzero scalars $\{q\} \cup\left\{p_{i j} \mid 1 \leq i<j \leq n\right\}$, the quantum matrix algebra $M_{q, p_{i j}}(n)$ is generated by $\left\{x_{i j} \mid 1 \leq i, j \leq n\right\}$ subject to the relations

$$
\begin{aligned}
& x_{i t} x_{i s}=q p_{s t}^{-1} x_{i s} x_{i t} \quad \forall s<t, \\
& x_{j s} x_{i s}=q p_{i j} x_{i s} x_{j s} \quad \forall i<j, \\
& x_{i t} x_{j s}=p_{s t}^{-1} p_{i j}^{-1} x_{j s} x_{i t} \quad \forall i<j, s<t, \\
& x_{i s} x_{j t}=p_{s t} p_{i j}^{-1} x_{j t} x_{i s}+p_{i j}^{-1}\left(q-q^{-1}\right) x_{j s} x_{i t} \quad \forall i<j, s<t .
\end{aligned}
$$

By [AST, 2] $M_{q, p_{i j}}(n)$ is a left and right noetherian domain. It is also an iterated Ore extension of the base field $k$. If we define $\operatorname{deg}\left(x_{i j}\right)=e_{n(i-1)+j} \in \mathbb{N}^{n^{2}}$, then the ordered monomials form a $k$-linear basis of $M_{q, p_{i j}}(n)$. By using the relations (7.4.1) one can check that $M_{q, p_{i j}}(n)$ is an $\mathbb{N}^{n^{2}}$-filtered algebra such that the associated 
graded algebra is isomorphic to the skew polynomial algebra generated by $\left\{x_{i j} \mid 1 \leq\right.$ $i, j \leq n\}$ subject to the relations

$$
\begin{aligned}
x_{i t} x_{i s} & =q p_{s t}^{-1} x_{i s} x_{i t} & & \forall s<t, \\
x_{j s} x_{i s} & =q p_{i j} x_{i s} x_{j s} & & \forall i<j, \\
x_{i t} x_{j s} & =p_{s t}^{-1} p_{i j}^{-1} x_{j s} x_{i t} & & \forall i<j, s<t, \\
x_{i s} x_{j t} & =p_{s t} p_{i j}^{-1} x_{j t} x_{i s} & & \forall i<j, s<t .
\end{aligned}
$$

Since the relations (7.4.1) are quadratic, $M_{q, p_{i j}}(n)$ is connected $\mathbb{N}$-graded and generated by elements $\left\{x_{i j} \mid 1 \leq i, j \leq n\right\}$ of degree 1. By [AST, 1] $M_{q, p_{i j}}(n)$ and the polynomial algebra of $n^{2}$ variables have the same Hilbert function. As a consequence, $\operatorname{GKdim}\left(M_{q, p_{i j}}(n)\right)=n^{2}$. By Theorem 7.3(3), $M_{q, p_{i j}}(n)$ is Tdegstable.

The quantum determinant $D$ of $M_{q, p_{i j}}(n)$ is a normal element of degree $n$. Let $\sigma$ be the automorphism of $M_{q, p_{i j}}(n)$ determined by $a D=D a^{\sigma}$. An automorphism $\alpha$ of $A$ is said to be locally algebraic if for every $a \in A$ the set $\left\{\alpha^{n}(a)\right\}$ is contained in a finite dimensional subspace of $A$ [LMO, p. 208]. Since $M_{q, p_{i j}}(n)$ is $\mathbb{N}$-graded locally finite and $D$ is homogeneous, the automorphism $\sigma$ is locally algebraic. The quantum group $G L_{q, p_{i j}}(n)$ is defined to be the localization $M_{q, p_{i j}}(n)\left[D^{-1}\right]$. By [LMO, Th. 2], $\operatorname{GKdim}\left(G L_{q, p_{i j}}(n)\right)=\operatorname{GKdim}\left(M_{q, p_{i j}}(n)\right)$. By definition, the quotient algebras of $M_{q, p_{i j}}(n)$ and $G L_{q, p_{i j}}(n)$ are the same. Therefore

$$
\operatorname{Tdeg}\left(Q\left(G L_{q, p_{i j}}(n)\right)\right)=\operatorname{Tdeg}\left(Q\left(M_{q, p_{i j}}(n)\right)\right)=n^{2}=\operatorname{GKdim}\left(G L_{q, p_{i j}}(n)\right) .
$$

By Proposition 3.5(2), $G L_{q, p_{i j}}(n)$ is Tdeg-stable.

Example 7.5. The quantum Weyl algebras $A_{n}\left(q, p_{i j}\right)$ are Tdeg-stable.

For details of $A_{n}\left(q, p_{i j}\right)$ see [GZ]. Given a set of nonzero scalars $\{q\} \cup\left\{p_{i j} \mid 1 \leq\right.$ $i<j \leq n\}$, the quantum Weyl algebra $A_{n}\left(q, p_{i j}\right)$ is generated by $\left\{x_{1}, \cdots, x_{n}\right\} \cup$ $\left\{\partial_{1}, \cdots, \partial_{n}\right\}$ subject to the relations

$$
\begin{aligned}
x_{i} x_{j} & =p_{i j} q x_{j} x_{i} & & \forall i<j, \\
\partial_{i} \partial_{j} & =p_{i j} q^{-1} \partial_{j} \partial_{i} & & \forall i<j, \\
\partial_{i} x_{j} & =p_{i j}^{-1} q x_{j} \partial_{i} & & \forall i \neq j, \\
\partial_{i} x_{i} & =1+q^{2} x_{i} \partial_{i}+\left(q^{2}-1\right) \sum_{j>i} x_{j} \partial_{j} & & \forall i .
\end{aligned}
$$

Define $\operatorname{deg}\left(x_{i}\right)=n+1-i$ and $\operatorname{deg}\left(\partial_{i}\right)=2 n+1-i$. By [GZ, 1.5] and easy computations, $A_{n}\left(q, p_{i j}\right)$ is a filtered skew polynomial algebra, and by [GZ, 3.10.3], $\operatorname{GKdim}\left(A_{n}\left(q, p_{i j}\right)\right)=2 n$. By Theorem 7.3(3), $A_{n}\left(q, p_{i j}\right)$ is Tdeg-stable.

Example 7.6. The quantum universal enveloping algebra $U_{q}\left(s l_{2}\right)$ is Tdeg-stable.

There are two versions of $U_{q}\left(s l_{2}\right)$. The first version is Jimbo's quantum group $U_{q}\left(s l_{2}\right)[\mathrm{Ji}]$, which is an algebra generated by $\left\{e, f, h, h^{-1}\right\}$ subject to the relations

$$
\begin{aligned}
h h^{-1} & =1=h^{-1} h, \\
h e & =q^{2} e h, \\
h f & =q^{-2} f h, \\
e f-f e & =\frac{h-h^{-1}}{q-q^{-1}},
\end{aligned}
$$


where $q \in k-\{0,-1,1\}$. The quantum algebra $U_{q}\left(s l_{2}\right)$ can be expressed as an Ore extension $k_{q^{-2}}[h, e][f, \sigma, \delta]$, where $\sigma$ is an automorphism sending $h$ to $q^{-2} h$ and $e$ to $e$ and where $\delta$ is a $\sigma$-derivation sending $h$ to 0 and $e$ to $\frac{h-h^{-1}}{q-q^{-1}}$. Let $f^{\prime}=f h$. Then the relations (7.6.1.) become

$$
\begin{aligned}
h e & =q^{2} e h, \\
h f^{\prime} & =q^{-2} f^{\prime} h, \\
e f^{\prime}-q^{-2} f^{\prime} e & =\frac{h^{2}-1}{q-q^{-1}} .
\end{aligned}
$$

It is easy to see that the algebra, say $U$, generated by $\left\{e, f^{\prime}, h\right\}$ subject to relations (7.6.2) is a filtered skew polynomial algebra with $\operatorname{deg}(h)=(1,0,0), \operatorname{deg}(e)=(0,1,0)$ and $\operatorname{deg}(f)=(0,0,1)$. By a direct computation we see that $\operatorname{GKdim}(U)=3$. Hence by Theorem 7.3(3), $U$ is Tdeg-stable. Now $U_{q}\left(s l_{2}\right)$ is a localization $U\left[h^{-1}\right]$, and, by $[\mathrm{LMO}, \mathrm{Th} .2], \operatorname{GKdim}\left(U_{q}\left(s l_{2}\right)\right)=\operatorname{GKdim}(U)=3$. Hence

$$
\operatorname{Tdeg}\left(Q\left(U_{q}\left(s l_{2}\right)\right)\right)=\operatorname{Tdeg}(Q(U))=3=\operatorname{GKdim}\left(U_{q}\left(s l_{2}\right)\right) .
$$

Therefore $U_{q}\left(s l_{2}\right)$ is Tdeg-stable.

The second version of the quantum universal enveloping algebra of $s l_{2}$ was studied in [JZ]. Given $q \in k-\{0\}$, the quantum universal enveloping algebra $U_{q}^{\prime}\left(s l_{2}\right)$ is generated by $\{e, f, h\}$ subject to the relations

$$
\begin{aligned}
q h e-e h & =2 e, \\
h f-q f h & =-2 f, \\
e f-q f e & =h+\frac{1-q}{4} h^{2} .
\end{aligned}
$$

Define $\operatorname{deg}(h)=(1,0,0), \operatorname{deg}(e)=(0,1,0)$ and $\operatorname{deg}(f)=(0,0,1)$. Then this $U_{q}^{\prime}\left(s l_{2}\right)$ is a filtered skew polynomial algebra of three variables. By [JZ, 3.7], $\operatorname{GKdim}\left(U_{q}^{\prime}\left(s l_{2}\right)\right)=3$, and by Theorem 7.3(3), $U_{q}^{\prime}\left(s l_{2}\right)$ is Tdeg-stable.

\section{Graded Algebras}

In this section we will discuss some properties of Tdeg concerning $G$-graded algebras. Except for Theorem 8.10, we assume that $G$ is an ordered semigroup. Note that every finitely generated torsion-free nilpotent group is ordered [Pa, 13.1.6]. First let us state a nice result of Lorenz [Lo, 2.2] without proof. For the definitions of twisted group algebra and crossed product see [MR, 1.5.9].

Theorem 8.1 (Lorenz). Let $G$ be a finitely generated torsion-free nilpotent group of class at most 2 and let $k * G$ be a twisted group algebra. Then $k * G$ is Tdeg-stable.

Proposition 8.2. Let $G$ be an ordered semigroup and let $A$ be a G-graded domain. Then

$$
\operatorname{Tdeg}(A)=\sup _{V} \inf _{b} \operatorname{GKdim}(k[b V]),
$$

where $V$ ranges over all graded subframes of $A$ and $b$ ranges over all non-zero homogeneous elements of $A$.

Proof. By Example 6.1, the leading-term map $\nu$ is a valuation from $A$ to $A$. By the proof of Theorem $6.7(2)$, we have $\operatorname{GKdim}(k[a V]) \geq \operatorname{GKdim}(k[\nu(a) \nu(V)])$. If we let 
$V=\nu_{h}(W)$, then $V=\nu_{h}(V)$. Hence $I_{V}(A)=\inf _{\nu(a)} \operatorname{GKdim}(k[\nu(a) V])$. Therefore the statement follows from (6.6.2).

For every graded subframe $V$ of $A$ we denote

$$
I_{V}^{g r}(A)=\inf _{b} \operatorname{GKdim}(k[b V])
$$

where $b$ ranges over the homogeneous regular elements of $A$. By the proof of Proposition 8.2, if $G$ is an ordered semigroup and $A$ is a $G$-graded domain, then $I_{V}^{g r}(A)=I_{V}(A)$.

If $G$ is a group, a $G$-graded division algebra is isomorphic to a crossed product $D * G^{\prime}$ for some division algebra $D$ and some subgroup $G^{\prime} \subset G$ [NV1, I4.2.3]. The next proposition is a consequence of Theorem 6.7(3), which is also a consequence of $[\mathrm{Lo}, 1.2]$.

Proposition 8.3. Suppose that $G$ is an ordered group and $D$ is a division algebra. Let $A=D * G$ be a crossed product of $D$ by $G$ and let $Q(A)$ be the quotient algebra of $A$. Then $\operatorname{Tdeg}(Q(A))=\operatorname{Tdeg}(A)$.

Proof. Since $G$ is ordered, $A$ is a domain. By Example 6.1, the leading-term map $\nu$ is a valuation from $A$ to $A$. For every nonzero element $x \in A, \nu(x)$ is invertible because $\nu(x)$ is homogeneous. By Theorem 6.7(3) and Proposition 3.1(3), $\operatorname{Tdeg}(Q(A))=\operatorname{Tdeg}(A)$.

The next example is an analogue of Example 7.1.

Example 8.4. Twisted group algebras and valuations.

Let $G$ be an ordered semigroup. Suppose $k * G$ is a twisted group algebra. We can identify $k * G$ with the group algebra $k[G]$ as a vector space and let $I d_{G}: k * G \longrightarrow$ $k[G]$ be the identity map of the vector space. Let $D$ be a domain, $A=D \otimes k * G$ and $B=D \otimes k[G]$. By Example 6.1, the leading-term map $\nu$ is a valuation from $A$ to $A$. It is easy to check that $\mu:=\left(i d_{D} \otimes I d_{G}\right) \circ \nu$ is a valuation from $A$ to $B$ (see Example 7.1 for a special case). Similarly, let $\nu_{1}$ be the leading-term map of $B=D \otimes k[G]$ defined in Example 6.1; then $\alpha:=\left(i d_{D} \otimes I d_{G}^{-1}\right) \circ \nu_{1}$ is a valuation from $B$ to $A$.

By using these valuations we can show the following proposition.

Proposition 8.5. Let $G$ be an ordered semigroup and let $D$ be a domain. Then

(1) $\operatorname{GKdim}(D \otimes k * G)=\operatorname{GKdim}(D \otimes k[G])$.

(2) $\operatorname{Tdeg}(D \otimes k * G)=\operatorname{Tdeg}(D \otimes k[G])$.

(3) Let $G$ be an ordered group. Then $\operatorname{Tdeg}(Q(D \otimes k * G))=\operatorname{Tdeg}(Q(D \otimes k[G]))$. As a consequence, if $D \otimes k[G]$ is Tdeg-stable, then so is $D \otimes k * G$.

(4) $\mathrm{Tdeg}(D \otimes k * G) \leq \mathrm{T} \operatorname{deg}(D)+\mathrm{T} \operatorname{deg}(k * G)$.

Proof. (1) and (2). By Example 8.4, $\mu$ is a valuation from $A=D \otimes k * G$ to $B=$ $D \otimes k[G]$. It is easy to see that $A$ and $B$ are domains and $\mu(A)=B$. By Theorems 6.7(1) and 6.7(2), $\operatorname{GKdim}(A) \geq \operatorname{GKdim}(B)$ and $\operatorname{Tdeg}(A) \geq \operatorname{Tdeg}(B)$. Since $\alpha$ is a valuation from $B$ to $A$, we have $\operatorname{GKdim}(A) \leq \operatorname{GKdim}(B)$ and $\operatorname{Tdeg}(A) \leq \operatorname{Tdeg}(B)$. Therefore (1) and (2) are proven.

(3) A consequence of (2) and Proposition 8.3.

As a consequence of (1) and (3), if $D \otimes k[G]$ is Tdeg-stable, then $D \otimes k * G$ is Tdeg-stable. 
(4) By (2), it suffices to show $\operatorname{Tdeg}(D \otimes k[G]) \leq \operatorname{Tdeg}(D)+\operatorname{Tdeg}(k[G])$. By Proposition 8.2, only graded subframes $V$ and homogeneous elements $b$ need to be considered. For every homogeneous element $b \in D \otimes k[G]$, there is an element $c \in D$ and $g \in G$ such that $b=c \otimes g$. For every subframe $W \subset D \otimes k[G]$, there is a subframe $V \subset D$ and a finite subset $H \subset G$ such that $W \subset V H$. Hence

$$
\begin{aligned}
I_{W}(D \otimes k[G]) & \leq I_{V H}^{g r}(D \otimes k[G])=\inf _{c \otimes g} \operatorname{GKdim}(k[(c \otimes g) V H]) \\
& \leq \inf _{c \otimes g} \operatorname{GKdim}(k[c V] \otimes k[g H]) \\
& \leq \inf _{c \otimes g}\{\operatorname{GKdim}(k[c V])+\operatorname{GKdim}(k[g(k H)])\} \\
& =I_{V}(D)+I_{k H}^{g r}(k[G]) \leq \operatorname{Tdeg}(D)+\operatorname{Tdeg}(k[G]) .
\end{aligned}
$$

Since $W$ is arbitrary, $\operatorname{Tdeg}(D \otimes k[G]) \leq \operatorname{Tdeg}(D)+\operatorname{Tdeg}(k[G])$.

By using Proposition 8.2 we can also prove the following.

Proposition 8.6. Let $G$ be an ordered semigroup and let $N$ be a normal subsemigroup of $G$ such that $G / N$ is a finite group. Suppose that $A$ is a G-graded domain and let $A^{(N)}=\bigoplus_{g \in N} A_{g}$. Then $\operatorname{Tdeg}(A) \geq \operatorname{Tdeg}\left(A^{(N)}\right)$.

Proof. Let $V$ be a graded subframe of $A^{(N)}$. For every homogeneous element $b$ of $A, b^{n} \in A^{(N)}$ for some $n$ because $G / N$ is finite. Hence

$$
I_{V}^{g r}(A)=\inf _{b} \operatorname{GKdim}(k[b V]) \geq \inf _{b^{n}} \operatorname{GKdim}\left(k\left[b^{n} V\right]\right) \geq I_{V}^{g r}\left(A^{(N)}\right) .
$$

Since $V$ is arbitrary, $\operatorname{Tdeg}(A) \geq \operatorname{Tdeg}\left(A^{(N)}\right)$.

In the next example valuations are not defined by filtrations on algebras.

Example 8.7. Other valuations of graded algebras.

Let $G$ be an ordered semigroup and let $A$ be a $G$-graded domain. Suppose that $I$ is an ideal of $A$ containing no homogeneous elements. Let $f$ be the canonical algebra homomorphism from $A$ to its factor algebra $A / I$. It is easy to see that $f \circ \nu$ is a valuation from $A$ to $A / I$, where $\nu$ is the leading-term map of $A$. This kind of valuation satisfies $(\mathrm{v} 1,2,3)$ and $(\mathrm{v} 4)^{\prime}$ but not $(\mathrm{v} 4)$. Here are two special cases.

(1) Let $A$ be a domain and let $A[G]$ be the group algebra. Let $N$ be a normal subgroup and let $I$ be the ideal generated by $\{g-1 \mid g \in N\}$. Then $I$ contains no homogeneous elements of $A$ and $A / I \cong A[G / N]$.

(2) Let $A$ be a $\mathbb{Z}$-graded domain and let $x$ be a central element of degree not zero. Then the ideal $I$ generated by $1-x$ contains no homogeneous elements.

Example 8.7 leads to the following proposition.

Proposition 8.8. Let $G$ be an ordered semigroup and let $A$ be a $G$-graded domain. Suppose that $I$ is an ideal of $A$ containing no homogeneous elements and suppose that the images of nonzero homogeneous elements are regular in $A / I$. Then $\operatorname{Tdeg}(A) \geq \operatorname{Tdeg}(A / I)+1$.

Proof. Let $f$ be the canonical algebra homomorphism from $A$ to $A / I$. Since $f$ is surjective, for every subframe $V \subset A / I$, there is a graded subframe $W \subset A$ such 
that $f(W) \supset V$. Replacing $W$ by a larger subframe if necessary, we may assume $W \cap I \neq 0$. Hence for every nonzero homogeneous element $b, k[b W] \cap I \neq 0$. Thus

$$
\operatorname{GKdim}(k[b W]) \geq \operatorname{GKdim}(k[f(b) f(W)])+1 \geq I_{f(W)}(A / I)+1 \geq I_{V}(A / I)+1 .
$$

Since $b$ and $V$ are arbitrary, $\operatorname{Tdeg}(A) \geq \operatorname{Tdeg}(A / I)+1$.

Mimicking the proof of Proposition 8.8, the following can be obtained.

Proposition 8.9. If $f: A \longrightarrow B$ is an algebra epimorphism such that $f(b)$ is regular for every regular element $b$ of $A$. Then $\operatorname{Tdeg}(A) \geq \operatorname{Tdeg}(B)$.

Theorem 8.10. If $G$ is a finite group, then $\operatorname{Tdeg}(A[G])=\operatorname{Tdeg}(A)$.

Proof. Let $f: A[G] \longrightarrow A$ be the map defined by sending $g$ to 1 for all $g \in G$. Then $f$ is an algebra epimorphism. Suppose $b=\sum a_{g} \otimes g$ is a regular element of $A[G]$. We claim that $f(b)=\sum_{g} a_{g}$ is regular in $A$. If not, say $f(b)$ not right regular, then there is an element $c \in A$ such that $\left(\sum_{g} a_{g}\right) c=0$. Then $b\left(\sum_{g} c \otimes g\right)=$ $\left(\sum_{g} a_{g}\right) c \otimes\left(\sum_{g} g\right)=0$. This contradicts the regularity of $b$. By Proposition 8.9, $\operatorname{Tdeg}(A[G]) \geq \operatorname{Tdeg}(A)$.

Conversely, consider a subframe $W \subset A[G]$. We may assume $W=V G$ for some subframe $V$ of $A$. Every regular element $b$ of $A$ is also regular in $A[G]$. It is easy to see that

$$
k[b V] \subset k[b V G] \subset k[b V][G] .
$$

Hence $\operatorname{GKdim}(k[b V G])=\operatorname{GKdim}(k[b V])$ and

$$
I_{V G}(A[G]) \leq \inf _{b \in A} \operatorname{GKdim}(k[b V G]) \leq \inf _{b \in A} \operatorname{GKdim}(k[b V])=I_{V}(A) .
$$

Since $W=V G$ is arbitrary, $\operatorname{Tdeg}(A[G]) \leq \operatorname{Tdeg}(A)$.

\section{Polynomial Extensions}

In this section we will discuss the relationship between $\operatorname{Tdeg}(A[x, \sigma, \delta])$ and $\operatorname{Tdeg}(A)$ for some special $\sigma$ and $\delta$.

Given an automorphism $\sigma$ and a $\sigma$-derivation $\delta$ of $A$, the algebra $A[x, \sigma, \delta]$ is a polynomial extension of $A$ satisfying $a x=x \sigma(a)+\delta(a)$ for all $a \in A$ (see [MR, Sec. 1.2]). If $\delta=0$, then $x$ is a regular normal element and the Laurent polynomial algebra $A\left[x, x^{-1}, \sigma\right]$ is obtained by inverting the regular element $x$ in $A[x, \sigma]$ (see $[\mathrm{MR}$, Sec. 1.4]). By [LMO, Lemma 1], $\operatorname{GKdim}(A[x, \sigma]) \geq \operatorname{GKdim}(A)+1$. Since $A[x, \sigma]$ is a subalgebra of $A\left[x, x^{-1}, \sigma\right]$, we have

$$
\operatorname{GKdim}\left(A\left[x, x^{-1}, \sigma\right]\right) \geq \operatorname{GKdim}(A[x, \sigma]) \geq \operatorname{GKdim}(A)+1 .
$$

A natural filtration of $A[x, \sigma, \delta]$ is defined by $F_{n}=\left\{a_{0}+a_{1} x+\cdots a_{n} x^{n} \mid a_{i} \in A\right\}$ for all $n \in \mathbb{N}$, and the associated graded algebra of $A[x, \sigma, \delta]$ is isomorphic to $A[x, \sigma]$. By $[\mathrm{MR}, 8.3 .20]$,

$$
\operatorname{GKdim}(A[x, \sigma, \delta]) \geq \operatorname{GKdim}(A[x, \sigma]) \geq \operatorname{GKdim}(A)+1 .
$$

There are examples with $\operatorname{GKdim}(A[x, \sigma])>\operatorname{GKdim}(A)+1$ (see $[\mathrm{MR}, 8.2 .16]$ or Example 2.3(1)) and with $\operatorname{GKdim}(A[x, \delta])>\operatorname{GKdim}(A)+1$ [KL, 3.9]. However in some special cases the equality holds. A subspace $V \subset A$ is called $\sigma$-stable (or $\delta$-stable), if $\sigma(V) \subset V$ (or $\delta(V) \subset V$ ). If every subframe $W \subset A$ is contained in a $\sigma$-stable subframe $V$, then $\sigma$ is called locally algebraic (this definition is equivalent to the one given in Example 7.4). 
Lemma 9.1. Let $\sigma$ be an automorphism of an algebra $A$ and let $\delta$ be a $\sigma$-derivation of $A$.

(1) If $\sigma$ is locally algebraic, then

$$
\operatorname{GKdim}(A[x, \sigma])=\operatorname{GKdim}\left(A\left[x, x^{-1}, \sigma\right]\right)=\operatorname{GKdim}(A)+1 .
$$

(2) If every subframe $W$ of $A$ is contained in a $\sigma$-stable subframe $V$ such that the subalgebra $k[V]$ is $\delta$-stable, then

$$
\operatorname{GKdim}(A[x, \sigma, \delta])=\operatorname{GKdim}(A)+1 .
$$

Proof. (1) is [LMO, Prop. 1]. (2) is an analogue of [KL, 3.5]; we can copy the proof of that result without change by starting with a $\sigma$-stable subframe $V$ of $A$.

If $\sigma$ is the identity and $\delta$ is zero, Tdeg behaves like many other invariants.

Theorem 9.2. Let $A$ be a domain. Then

$$
\operatorname{Tdeg}\left(A\left[x, x^{-1}\right]\right)=\operatorname{Tdeg}(A[x])=\operatorname{Tdeg}(A)+1 .
$$

(2) $A$ is Tdeg-stable if and only if $A[x]$ (or $A\left[x, x^{-1}\right]$ ) is Tdeg-stable.

Proof. (1) By Proposition 3.1(3), $\operatorname{Tdeg}\left(A\left[x, x^{-1}\right]\right) \leq \operatorname{Tdeg}(A[x])$. Hence we need to show:

(a) $\operatorname{Tdeg}\left(A\left[x, x^{-1}\right]\right) \geq \operatorname{Tdeg}(A)+1$, and

(b) $\operatorname{Tdeg}(A[x]) \leq \operatorname{Tdeg}(A)+1$.

(a) Since $A$ is a domain, $A\left[x, x^{-1}\right]$ is a $\mathbb{Z}$-graded domain with $\operatorname{deg}(A)=0$ and $\operatorname{deg}(x)=1$. The ideal $I$ generated by $(x-1)$ contains no homogeneous elements and $A / I \cong A$ is a domain. By Proposition 8.8, $\operatorname{Tdeg}\left(A\left[x, x^{-1}\right]\right) \geq \operatorname{Tdeg}(A)+1$.

(b) Since $A[x]=A \otimes k[x]$ and since $k[x]$ is the semigroup algebra $k[\mathbb{N}]$, by Proposition 8.5(4),

$$
\operatorname{Tdeg}(A[x]) \leq \operatorname{Tdeg}(A)+\operatorname{Tdeg}(k[x])=\operatorname{Tdeg}(A)+1 .
$$

(2) First note that $A[x]$ is Tdeg-stable if and only if $A\left[x, x^{-1}\right]$ is Tdeg-stable. Let $Q$ be the quotient division algebra of $A$. By Proposition 8.3 and (1),

$$
\operatorname{Tdeg}\left(Q\left(A\left[x, x^{-1}\right]\right)\right)=\operatorname{Tdeg}\left(Q\left[x, x^{-1}\right]\right)=\operatorname{Tdeg}(Q)+1 .
$$

Combining this with [MR, 8.2.15] (or Lemma 9.1(1)) we obtain

$$
\operatorname{Tdeg}\left(Q\left(A\left[x, x^{-1}\right]\right)\right)-\operatorname{GKdim}\left(A\left[x, x^{-1}\right]\right)=\operatorname{Tdeg}(Q)-\operatorname{GKdim}(A) .
$$

Therefore by Proposition 3.5(2) $A$ is Tdeg-stable if and only if $A\left[x, x^{-1}\right]$ is Tdegstable.

The next propositions are partial results about $\operatorname{Tdeg}(A[x, \sigma, \delta])$ when $\sigma$ is not the identity and/or $\delta$ is not zero. 
Proposition 9.3. Let $A$ be a domain, $\sigma$ an automorphism of $A$ and $\delta$ a $\sigma$ derivation. Then

(1) The following inequalities hold:

$$
\operatorname{Tdeg}(A[x, \sigma, \delta]) \geq \operatorname{Tdeg}(A[x, \sigma])
$$

and

$$
\operatorname{Tdeg}(Q(A[x, \sigma, \delta])) \geq \operatorname{Tdeg}\left(Q(A)\left[x, x^{-1}, \sigma\right]\right) .
$$

(2) Suppose that every subframe $W$ of $A$ is contained in a $\sigma$-stable subframe $V$ such that the subalgebra $k[V]$ is $\delta$-stable. If $A[x, \sigma]$ is Tdeg-stable, then $A[x, \sigma, \delta]$ is Tdeg-stable and $\operatorname{Tdeg}(A[x, \sigma])=\operatorname{Tdeg}(A[x, \sigma, \delta])$.

Proof. (1) Define a leading-term map $\nu: A[x, \sigma, \delta] \longrightarrow A[x, \sigma]$ by

$$
\nu\left(\sum_{i=0}^{n} a_{i} x^{i}\right)=a_{n} x^{n}
$$

where $a_{n}$ is the nonzero coefficient of the highest degree term of $\sum_{i=0}^{n} a_{i} x^{i}$. Hence $\nu$ is a valuation and $\nu(A[x, \sigma, \delta])=A[x, \sigma]$. By Theorem 6.7(2), $\operatorname{Tdeg}(A[x, \sigma, \delta]) \geq$ $\operatorname{Tdeg}(A[x, \sigma])$. By Proposition 6.5, the valuation $\nu$ can be extended to a valuation from $Q(A[x, \sigma, \delta])$ to $Q(A)\left[x, x^{-1}, \sigma\right]$ and by Theorem $6.7(2)$,

$$
\operatorname{Tdeg}(Q(A[x, \sigma, \delta])) \geq \operatorname{Tdeg}\left(Q(A)\left[x, x^{-1}, \sigma\right]\right) .
$$

(2) By the proof of (1), there is a valuation from $A[x, \sigma, \delta]$ to $A[x, \sigma]$. By $\operatorname{Lemma} 9.1(2), \operatorname{GKdim}(A[x, \sigma, \delta])=\operatorname{GKdim}(A[x, \sigma])$ and by Theorem $6.8, A[x, \sigma]$ being Tdeg-stable implies that $A[x, \sigma, \delta]$ is Tdeg-stable. Therefore $\operatorname{Tdeg}(A[x, \sigma])=$ $\operatorname{Tdeg}(A[x, \sigma, \delta])$.

Proposition 9.4. Let $A$ be a domain, $\sigma$ an automorphism of $A$ of finite order and $\delta$ a $\sigma$-derivation of $A$. Then

$$
\operatorname{Tdeg}(A[x, \sigma, \delta]) \geq \operatorname{Tdeg}\left(A\left[x, x^{-1}, \sigma\right]\right) \geq \operatorname{Tdeg}(A)+1 .
$$

(2) Suppose that every subframe $W \subset A$ is contained in a $\sigma$-stable subframe $V$ such that the subalgebra $k[V]$ is $\delta$-stable. If $A$ is Tdeg-stable, then $A\left[x, x^{-1}, \sigma\right]$ and $A[x, \sigma, \delta]$ are Tdeg-stable.

Proof. (1) By Proposition 9.3(1),

$$
\operatorname{Tdeg}(A[x, \sigma, \delta]) \geq \operatorname{Tdeg}(A[x, \sigma]) \geq \operatorname{Tdeg}\left(A\left[x, x^{-1}, \sigma\right]\right) .
$$

Since $\sigma$ has finite order, say $n$, we have $\left(A\left[x, x^{-1}, \sigma\right]\right)^{(n)} \cong A\left[z, z^{-1}\right]$. By Proposition 8.6, and Theorem 9.2(1),

$$
\operatorname{Tdeg}\left(A\left[x, x^{-1}, \sigma\right]\right) \geq \operatorname{Tdeg}\left(\left(A\left[x, x^{-1}, \sigma\right]\right)^{(n)}\right)=\operatorname{Tdeg}\left(A\left[z, z^{-1}\right]\right)=\operatorname{Tdeg}(A)+1 .
$$

Thus we have proved (1).

(2) By Proposition 9.3(1) and (1) ( $\sigma$ is also an automorphism of $Q(A)$ of finite order),

$$
\operatorname{Tdeg}(Q(A[x, \sigma, \delta])) \geq \operatorname{Tdeg}\left(Q(A)\left[x, x^{-1}, \sigma\right]\right) \geq \operatorname{Tdeg}(Q(A))+1 .
$$

Since $A$ is Tdeg-stable, and by Lemma 9.1(2),

$$
\operatorname{Tdeg}(Q(A))+1=\operatorname{GKdim}(A)+1=\operatorname{GKdim}(A[x, \sigma, \delta]) .
$$

Hence by Proposition 3.5(2) $A[x, \sigma, \delta]$ is Tdeg-stable. A similar proof shows that $A\left[x, x^{-1}, \sigma\right]$ is Tdeg-stable. 
Proposition 9.5. Let $A$ be a commutative domain and let $\sigma$ be a locally algebraic automorphism of $A$. Then $\operatorname{Tdeg}\left(A\left[x, x^{-1}, \sigma\right]\right)=\operatorname{GKdim}(A)+1$.

Proof. By Proposition 3.1(3), $\operatorname{Tdeg}\left(A\left[x, x^{-1}, \sigma\right]\right) \leq \operatorname{Tdeg}(A[x, \sigma])$. It suffices to show that

(a) $\operatorname{Tdeg}\left(A\left[x, x^{-1}, \sigma\right]\right) \geq \operatorname{Tdeg}(A)+1=\operatorname{GKdim}(A)+1$, and

(b) $\operatorname{Tdeg}(A[x, \sigma]) \leq \operatorname{Tdeg}(A)+1=\operatorname{GKdim}(A)+1$.

(a) Pick a $\sigma$-stable subframe $V \subset A$ such that $\operatorname{GKdim}(k[V])=\operatorname{GKdim}(A)$. Let $V^{\prime}=V+x V+x^{-1} V$. Then $x V^{\prime}=V^{\prime} x$. By Proposition 8.2, $I_{V^{\prime}}\left(A\left[x, x^{-1}, \sigma\right]\right)=$ $\inf _{b} \operatorname{GKdim}\left(k\left[b V^{\prime}\right]\right)$, where $b$ ranges over nonzero homogeneous elements. Hence $b=b_{1} x^{s}$ for some integer $s$ and some $b_{1} \in A$. It is easy to see that $b V^{\prime} \supset b_{1} V x^{i}$ for some $i \neq 0$. Thus

$$
\begin{aligned}
\operatorname{dim}\left(\left(k+b V^{\prime}\right)^{n}\right) & \geq \operatorname{dim}\left(\left(k+b_{1} x^{i} V\right)^{n}\right) \\
& =\operatorname{dim}\left\{k \oplus V\left(b_{1} x^{i}\right) \oplus(V)^{2}\left(b_{1} x^{i}\right)^{2} \oplus \cdots \oplus(V)^{n}\left(b_{1} x^{i}\right)^{n}\right\} \\
& =\sum_{l=0}^{n} \operatorname{dim}\left(V^{l}\right) .
\end{aligned}
$$

This implies that

$$
\operatorname{GKdim}\left(k\left[b V^{\prime}\right]\right) \geq \operatorname{GKdim}(k[V])+1=\operatorname{GKdim}(A)+1 .
$$

Therefore $\operatorname{Tdeg}\left(A\left[x, x^{-1}, \sigma\right]\right) \geq \operatorname{GKdim}(A)+1$.

(b) Since $\sigma$ is locally algebraic, by Lemma $9.1(1) \operatorname{GKdim}(A[x, \sigma])=\operatorname{GKdim}(A)+$ 1. Hence

$$
\operatorname{Tdeg}(A[x, \sigma]) \leq \operatorname{GKdim}(A[x, \sigma])=\operatorname{GKdim}(A)+1 .
$$

It can be shown that $\operatorname{Tdeg}\left(A\left[x, x^{-1}, \sigma\right]\right) \geq \operatorname{GKdim}(A)+1$ for every automorphism $\sigma$ of a commutative domain $A$, but the proof is more complicated (see [Z2]). By Lorenz's example (see the second part of Example 2.3(1)) inequality cannot be replaced by equality. Theorem 9.2, Propositions 9.4, 9.5 and Example 2.3(1) suggest the following conjecture.

Conjecture 9.6. Let $D$ be a domain and let $\sigma$ be an automorphism of $D$. Then

$$
\operatorname{Tdeg}\left(D\left[z, z^{-1}, \sigma\right]\right) \geq \operatorname{Tdeg}(D)+1 .
$$

If Conjecture 9.6 holds, then we can prove the conjecture stated in the introduction. Let $A$ be an $\mathbb{N}$-graded domain of finite GKdim. Then $A$ is an Ore domain. It is easy to check that $A$ is also a graded Ore domain. The graded quotient algebra $Q_{g r}(A)$ is then a strongly graded algebra and the degree zero part $D:=Q_{0}$ is a division algebra. There is an automorphism $\sigma$ of $D$ such that $Q_{g r}(A)=D\left[z, z^{-1}, \sigma\right]$ with $x$ having minimal positive degree in $Q_{g r}(A)$.

Proposition 9.7. Suppose Conjecture 9.6 is true. Let $A$ be a finitely generated connected graded domain of GKdim $d$, with graded quotient algebra $D\left[z, z^{-1}, \sigma\right]$. Then $\operatorname{Tdeg}(D) \leq d-1$. If, moreover, $\operatorname{GKdim}(A)=d=3$, then $\operatorname{Tdeg}(D)=2$.

Proof. By Proposition 3.1(3),

$$
\operatorname{Tdeg}\left(D\left[z, z^{-1}, \sigma\right]\right) \leq \operatorname{Tdeg}(A) \leq \operatorname{GKdim}(A)=d .
$$


If Conjecture 9.6 holds, then $\operatorname{Tdeg}(D) \leq d-1$.

Suppose now $\operatorname{GKdim}(A)=d=3$. Hence $\operatorname{Tdeg}(D) \leq 2$. If $\operatorname{Tdeg}(D)<2$, then by Proposition $2.4 \operatorname{Tdeg}(D) \leq 1$. By Theorem 4.3, $\operatorname{GKdim}(D) \leq 1$. By [ASta, 1.15] $D$ is finitely generated as a division algebra. Then by [SSW], $D$ is a finite module over its center $Z$ and $Z$ is a finitely generated field of transcendence degree $\leq 1$. Finally by $[\mathrm{ASta}, 1.2] \operatorname{GKdim}(A) \leq 2$. That is a contradiction. Therefore $\operatorname{Tdeg}(D)=2$.

If $d \geq 4$, then $\operatorname{Tdeg}(D)$ may be less than $d-1$, as the following example shows.

Example 9.8. There is a connected graded and Tdeg-stable domain $A$ of GKdim 4 , with graded quotient algebra $D\left[z, z^{-1}, \sigma\right]$, such that $\operatorname{Tdeg}(D)=\operatorname{GKdim}(D)=2$.

Let $D$ be the field of rational functions $k(x, y), \sigma$ the automorphism of $D$ defined by $\sigma(x)=x, \sigma(y)=x y$ (see Example 2.3(1)) and $B$ the graded algebra $D\left[z, z^{-1}, \sigma\right]$ with $\operatorname{deg}(z)=1$ and $\operatorname{deg}(D)=0$. Hence $\operatorname{GKdim}(D)=\operatorname{Tdeg}(D)=2$. It is easy to see that $B$ is a localization of the algebra $S:=k\left[x, x^{-1}, y, y^{-1}\right]\left[z, z^{-1}, \sigma\right]$ defined in Example 2.3(1). By Example 2.3(1), $S$ is isomorphic to a group algebra $k[G]$ where $G=\langle y, z| x=[y, z]$ central $\rangle$ is a torsion free nilpotent group of class 2. By Lorenz's Theorem [Theorem 8.1], $S=k[G]$ is Tdeg-stable. By Proposition 3.5(1), $\operatorname{Tdeg}(B)=\operatorname{Tdeg}(k[G])=4$. Let $A$ be the graded subalgebra of $B$ generated by $\{1, z, x z, y z, x y z\}$. Hence $A$ is a connected graded algebra which is also a subalgebra of $k[G]$. Now the graded quotient algebra of $A$ is $B$. As a consequence, the (ungraded) quotient algebra of $A$ is equal to the (ungraded) quotient algebra of $B$ (and of $k[G]$ ). By Proposition 3.5(4), $A$ is Tdeg-stable and $\operatorname{GKdim}(A)=\operatorname{GKdim}(k[G])=4$.

Conjecture 9.6 is also related to a question of Artin and Stafford. Artin and Stafford showed that if $A$ is a connected graded domain, then $\operatorname{GKdim}(A)$ cannot be strictly between 2 and 11/5 [ASta, 0.1]. It is natural to ask if any number between $11 / 5$ and 3 appears as GKdim of a connected graded domain. If Conjecture 9.6 holds, then it is easy to show that no number strictly between 2 amd 3 appears as GKdim or Tdeg of a finitely generated connected graded domain (see the proof of Proposition 9.7).

Without using Conjecture 9.6, we can prove a partial result stated in Theorem 1.3 .

Theorem 9.9. Let $A$ be an $\mathbb{N}$-graded locally finite domain of GKdim $d$, containing a normal element of degree $l>0$. Let $Q_{g r}(A)=D\left[x, x^{-1}, \sigma\right]$ be the graded quotient algebra of $A$. Suppose one of the following conditions holds:

(i) $A$ is right noetherian.

(ii) $A$ is generated by elements of degrees 0 and 1 .

Then $\operatorname{Tdeg}(D) \leq d-1$. Equality holds if $d=2,3$.

Proof. Under the condition (i) or (ii), $A$ is a finitely generated right $A^{(l)}$-module. Then, by $[\mathrm{KL}, 5.5], \operatorname{GKdim}(A)=\operatorname{GKdim}\left(A^{(l)}\right)$ and it is easily to check that $A^{(l)}$ satisfies (i) or (ii) respectively. As a consequence $A^{(l)}$ is an Ore domain. It is routine to check that $Q_{g r}(A)^{(l)}=Q_{g r}\left(A^{(l)}\right)$. Hence we may replace $A$ by $A^{(l)}$ and assume that the normal element, say $r$, has degree 1 . After twisting by an automorphism $\sigma$, the normal element $r$ becomes central in $A^{\sigma}[\mathrm{Z} 1,2.12]$. Under the condition (i) or (ii) we have $\operatorname{GKdim}(A)=\operatorname{GKdim}\left(A^{\sigma}\right)$ (see $[\mathrm{Z} 1,5.7]$ ) because $A$ and $A^{\sigma}$ are the same as a graded vector space. By $[\mathrm{Z} 1,5.4], Q_{g r}\left(A^{\sigma}\right)=\left(Q_{g r}(A)\right)^{\sigma}$. Thus the 
degree zero part $Q_{0}(A)$ of $Q_{g r}(A)$ is isomorphic to the degree zero part $Q_{0}\left(A^{\sigma}\right)$ of $Q_{g r}\left(A^{\sigma}\right)$. Hence $D$ is invariant under the twisting. Therefore, without loss of generality, we may assume that $A$ contains a central element $r$ of degree 1 .

Let $B$ be the localization $A\left[r^{-1}\right]$. Then by $[\mathrm{KL}, 4.2] \operatorname{GKdim}(B)=\operatorname{GKdim}(A)=$ $d$. By the definition of $B$ we have $B=B_{0}\left[r, r^{-1}\right]$ and $Q_{g r}(A)=Q_{g r}(B)$. Hence $D$ is isomorphic to the quotient division algebra $Q\left(B_{0}\right)$. By $[\mathrm{MR}, 8.2 .15], \operatorname{GKdim}\left(B_{0}\right)=$ $\operatorname{GKdim}(B)-1=d-1$. Therefore $\operatorname{Tdeg}(D) \leq \operatorname{GKdim}\left(B_{0}\right)=d-1$.

If $d=2$ or 3 , then $\operatorname{GKdim}\left(B_{0}\right)=1$ or 2 respectively. By Corollaries 4.2(4) and $4.4(3), \operatorname{Tdeg}(D)=1$ or 2 respectively.

If $d=3$, this is Theorem 1.3 stated in the introduction. If $D$ is commutative, then both Conjecture 9.6 and the conjecture stated in the introduction hold (details will be given in $[\mathrm{Z} 2])$.

\section{Regular Algebras}

In this section we will prove that Artin-Schelter regular algebras of dimension 3 and the Sklyanin algebra of dimension 4 are Tdeg-stable.

Let $\alpha, \beta$ and $\gamma$ be three scalars in $k$ not equal to 0,1 or -1 and satisfying

$$
\alpha+\beta+\gamma+\alpha \beta \gamma=0 .
$$

The Sklyanin algebra $S$ is generated by $\left\{x_{0}, x_{1}, x_{2}, x_{3}\right\}$ subject to the relations

$$
\begin{array}{ll}
x_{0} x_{1}-x_{1} x_{0}-\alpha\left(x_{2} x_{3}+x_{3} x_{2}\right)=0, & x_{0} x_{1}+x_{1} x_{0}-\left(x_{2} x_{3}-x_{3} x_{2}\right)=0, \\
x_{0} x_{2}-x_{2} x_{0}-\beta\left(x_{3} x_{1}+x_{1} x_{3}\right)=0, & x_{0} x_{2}+x_{2} x_{0}-\left(x_{3} x_{1}-x_{1} x_{3}\right)=0, \\
x_{0} x_{3}-x_{3} x_{0}-\gamma\left(x_{1} x_{2}+x_{2} x_{1}\right)=0, & x_{0} x_{3}+x_{3} x_{0}-\left(x_{1} x_{2}-x_{2} x_{1}\right)=0 .
\end{array}
$$

The algebra $S$ is a connected, graded, noetherian domain of GK-dimension, Krull dimension and global dimension 4 [SSta, 0.3]. By [SSta, 3.9] the elements

$$
\Omega_{1}=-x_{0}^{2}+x_{1}^{2}+x_{2}^{2}+x_{3}^{2} \quad \text { and } \quad \Omega_{2}=x_{1}^{2}+\frac{1+\alpha}{1-\beta} x_{2}^{2}+\frac{1-\alpha}{1+\gamma} x_{3}^{2}
$$

are central in $S$, and $S /\left(\Omega_{1}\right)$ and $S /\left(\Omega_{1}, \Omega_{2}\right)$ are domains of GKdim 3 and 2 respectively. Hence $\left\{\Omega_{1}, \Omega_{2}\right\}$ is a sequence of central regular elements. For more details see [SSta].

Proposition 10.1. Let $A$ be a domain and let $x \in A$ be a central element such that $\bigcap_{n} x^{n} A=\{0\}$. Suppose that $A /(x)$ is a domain. Then $\operatorname{Tdeg}(A) \geq \operatorname{Tdeg}(A /(x))+1$. If moreover $\operatorname{GKdim}(A)=\operatorname{GKdim}(A /(x))+1$ and $A /(x)$ is Tdeg-stable, then $A$ is Tdeg-stable.

Proof. By Proposition 6.10(2) and Theorem 9.2(1),

$$
\operatorname{Tdeg}(A) \geq \operatorname{Tdeg}(A /(x)[z])=\operatorname{Tdeg}(A /(x))+1 .
$$

Suppose that $\operatorname{GKdim}(A)=\operatorname{GKdim}(A /(x))+1$. Then

$$
\operatorname{GKdim}(A)=\operatorname{GKdim}(A /(x)[z]) .
$$

If $A /(x)$ is Tdeg-stable, by Theorem $9.2(2), A /(x)[z]$ is Tdeg-stable. Hence by Proposition 6.10(3), $A$ is Tdeg-stable.

Applying Proposition 10.1 to graded algebras, we have the following. 
Corollary 10.2. Let $A$ be a finitely generated connected graded domain and let $x$ be a central element of positive degree. Suppose $A /(x)$ is a domain. Then $\operatorname{Tdeg}(A) \geq$ $\operatorname{Tdeg}(A /(x))+1$. If moreover $A /(x)$ is Tdeg-stable, then $A$ is Tdeg-stable.

Proof. Since $A$ is graded and the degree of $x$ is positive, $\bigcap_{n} x^{n} A=\{0\}$. As a special case of Proposition 10.1, $\operatorname{Tdeg}(A) \geq \operatorname{Tdeg}(A /(x))+1$. Since $A:=\bigoplus_{i \geq 0} A_{i}$ is a finitely generated connected graded domain,

$$
\begin{aligned}
\operatorname{GKdim}(A) & =\varlimsup_{n \rightarrow \infty} \log _{n}\left(\sum_{i=0}^{n} \operatorname{dim} A_{i}\right) \\
& =\varlimsup_{n \rightarrow \infty} \log _{n}\left(\operatorname{dim} A_{n}\right)+1=\operatorname{GKdim}(A /(x))+1 .
\end{aligned}
$$

By Proposition 10.1, if $A /(x)$ is Tdeg-stable then $A$ is Tdeg-stable.

Corollary 10.3. The Sklyanin algebra $S$ of dimension 4 is Tdeg-stable.

Proof. Since $\left\{\Omega_{1}, \Omega_{2}\right\}$ is a sequence of central regular elements and $S /\left(\Omega_{1}, \Omega_{2}\right)$ is a domain of GKdim 2 which is Tdeg-stable [Corollary 4.4(3)], by Corollary 10.2, $S /\left(\Omega_{1}\right)$ and $S$ are Tdeg-stable.

The same proof works for any graded algebra $A$ with a central regular sequence $\left\{z_{i}\right\}_{i=1}^{m}$ such that $A /\left(z_{i}\right)$ is a Tdeg-stable (for example, $A /\left(z_{i}\right)$ is a domain of GKdim 2).

The Sklyanin algebra is Artin-Schelter regular in the following sense. A connected graded algebra $A$ is called Artin-Schelter regular (of dimension $d$ ) if

(as1) $A$ has finite global dimension $d$,

(as2) $A$ has finite GKdim,

(as3) $A$ is Gorenstein, namely $\operatorname{Ext}_{A}^{q}(k, A)$ is zero if $q \neq d$ and $k$ if $q=d$.

See $[\mathrm{AS}]$ and [Le] for more details. The Sklyanin algebra is an Artin-Schelter regular algebra of dimension 4 [SSta, 0.3]. The papers [AS], [ATV1,2] classify all Artin-Schelter regular algebras of dimension 3 which are generated in degree 1 . By [ATV1,2], a regular algebra $A$ of dimension 3 contains a normal element $z$ of positive degree such that $A /(z)$ is a graded domain of GKdim 2 . In some cases $z$ is central and, by Corollary 10.2, $A$ is Tdeg-stable. If $z$ is not central (but normal) we need a little more work to show that $A$ is Tdeg-stable.

Lemma 10.4. Let $A$ be a domain and $V$ a subframe of $A$ with $\operatorname{GKdim}(k[V]) \geq 1$.

(1) For every nonzero finite dimensional subspace $W$ of $A, \operatorname{dim}(W V)>\operatorname{dim}(W)$.

(2) Let $b_{i}$ be regular elements of $A$. Then $\operatorname{dim}\left(b_{1} V b_{2} V \cdots b_{n} V\right) \geq n+1$.

Proof. (1) Since $W V \supset W, \operatorname{dim}(W V)=\operatorname{dim}(W)$ implies that $W V=W$ and hence $W k[V]=W$. But $W$ contains a regular element, hence $\operatorname{dim}(k[V]) \leq \operatorname{dim}(W)$. This contradicts the fact that $\operatorname{GKdim}(k[V]) \geq 1$. Therefore $\operatorname{dim}(W V)>\operatorname{dim}(W)$.

(2) follows from (1) and induction.

Theorem 10.5. Let $D$ be a domain of GKdim $\geq 1$. Let $\sigma$ be an algebra automorphism of $D$ and let $\alpha$ be a graded algebra automorphism of $D\left[x, x^{-1}, \sigma\right]$ (with $\operatorname{deg}(D)=0$ and $\operatorname{deg}(x)=1)$. Then $\operatorname{Tdeg}\left(D\left[x, x^{-1}, \sigma\right]\left[y, y^{-1}, \alpha\right]\right) \geq 3$.

Proof. First note that $D\left[x, x^{-1}, \sigma\right]\left[y, y^{-1}, \alpha\right]$ is a $\mathbb{Z}^{2}$-graded algebra with $\operatorname{deg}(x)=$ $(1,0)$ and $\operatorname{deg}(y)=(0,1)$. Let $V$ be a subframe of $D$ such that $\operatorname{GKdim}(k[V]) \geq$ 
1. Then $V_{1}:=\sum_{s, t=-1}^{1} x^{s} y^{t} V$ is a subframe of $D\left[x, x^{-1}, \sigma\right]\left[y, y^{-1}, \alpha\right]$. For every nonzero homogeneous element $b_{1}=b x^{a} y^{b}$, we claim that $\operatorname{GKdim}\left(k\left[b_{1} V_{1}\right]\right) \geq 3$. By replacing $x$ by $x^{-1}$ and/or replacing $y$ by $y^{-1}$ if necessary, we may assume $b_{1} V_{1} \supset x^{i} y^{j} b(V+y V)$ and $i, j>0$. Let $W_{1}, W_{2}$ and $W$ denote $x^{i} y^{j} b V, x^{i} y^{j} b y V$ and $x^{i} y^{j} b(V+y V)$ respectively. Then $\left(k+b_{1} V_{1}\right)^{n} \supset(k+W)^{n}$ for all $n$. For every homogeneous element $c$ in $W$, the $\mathbb{Z}^{2}$-degree is $\operatorname{deg}(c)=(i, *)$. Hence

$$
(k+W)^{n}=\sum_{m=0}^{n} W^{m}=\bigoplus_{m=0}^{n} W^{m} .
$$

By definition, $W=W_{1} \oplus W_{2}$. Since $W_{1}^{s} W_{2}^{m-s}=\left(x^{i} y^{j} b V\right)^{s}\left(x^{i} y^{j} b y V\right)^{m-s}$ have different $\mathbb{Z}^{2}$-degrees for different $s$, we have

$$
W^{m} \supset \sum_{s=0}^{m} W_{1}^{s} W_{2}^{m-s}=\bigoplus_{s=0}^{m} W_{1}^{s} W_{2}^{m-s}
$$

By Lemma 10.4(2),

$$
\operatorname{dim}\left(W_{1}^{s} W_{2}^{m-s}\right)=\operatorname{dim}\left(\left(x^{i} y^{j} b V\right)^{s}\left(x^{i} y^{j} b y V\right)^{m-s}\right) \geq m+1 .
$$

Hence $\operatorname{dim}\left(W^{m}\right) \geq(m+1)(m+1)$ and

$$
\operatorname{dim}\left(\left(k+b_{1} V_{1}\right)^{n}\right) \geq \sum_{m=0}^{n}(m+1)^{2} \geq 1 / 3 n^{3} .
$$

Thus we have proved that $\operatorname{GKdim}\left(k\left[b_{1} V_{1}\right]\right) \geq 3$ for all nonzero homogeneous elements $b_{1}$. By Proposition 8.2, $\operatorname{Tdeg}\left(D\left[x, x^{-1}, \sigma\right]\left[y, y^{-1}, \alpha\right]\right) \geq 3$.

As a consequence of Theorem 10.5, we can prove the following.

Theorem 10.6. Let $A$ be a connected graded and finitely generated domain of GKdim 3, containing a normal element $z$ of positive degree such that $A /(z)$ is a domain. Then $A$ is Tdeg-stable.

Proof. Since $A$ is a connected graded finitely generated domain and $z$ is a normal element, by the proof of Corollary 10.2, $\operatorname{GKdim}(A)=\operatorname{GKdim}(A /(z))+1$. Hence $A /(z)$ is a connected graded finitely generated domain of GKdim 2. Then the graded quotient algebra $Q_{g r}(A /(z))$ is isomorphic to $D\left[x, x^{-1}, \sigma\right]$. By [ASta, 0.1] $D$ is a finitely generated PI division algebra with $\operatorname{GKdim}(D)=1$.

Since $z$ is a normal element of $A$, by Proposition 6.10(3),

$$
\operatorname{Tdeg}(A) \geq \operatorname{Tdeg}(A /(z)[y, \alpha])
$$

where $\alpha$ is determined by the normal element $z$. By Example 6.3, $\alpha$ is a graded algebra automorphism of $A /(z)$. Hence $A /(z)[y, \alpha]$ is a $\mathbb{Z}^{2}$-graded algebra with $\mathbb{Z}^{2}$-graded quotient algebra $D\left[x, x^{-1}, \sigma\right]\left[y, y^{-1}, \alpha\right]$. By Propositions 3.1(3) and 8.3 and Theorem 10.5,

$$
\begin{aligned}
\operatorname{Tdeg}\left(Q(A /(z))\left[y, y^{-1}, \alpha\right]\right) & \geq \operatorname{Tdeg}(Q(A /(z)[y, \alpha])) \\
& =\operatorname{Tdeg}\left(D\left[x, x^{-1}, \sigma\right]\left[y, y^{-1}, \alpha\right]\right) \geq 3 .
\end{aligned}
$$

Since there is a valuation from $A$ to $A /(x)[y, \alpha]$, by Proposition 6.5 , there is a valuation from $Q(A)$ to $Q(A /(z))\left[y, y^{-1}, \alpha\right]$. Therefore by Theorem 6.7(2),

$$
\operatorname{Tdeg}(Q(A)) \geq \operatorname{Tdeg}\left(Q(A /(z))\left[y, y^{-1}, \alpha\right]\right) \geq 3=\operatorname{GKdim}(A) .
$$

By Proposition 3.5(2), $A$ is Tdeg-stable. 
Corollary 10.7. Every 3-dimensional Artin-Schelter regular algebra generated in degree 1 is Tdeg-stable.

\section{REFERENCES}

[AS] M. Artin and W. Schelter, Graded algebras of global dimension 3, Adv. Math. 66 (1987), 171-216. MR 88k:16003

[AST] M. Artin, W. Schelter and J. Tate, Quantum deformations of $G L_{n}$, Comm. Pure Appl. Math. 64 (1991), 879-895. MR 92i:17014

[ASta] M. Artin and J. T. Stafford, Noncommutative graded domains with quadratic growth, Invent. Math. 122 (1995), 231-276. CMP 96:3

[ATV1] M. Artin, J. Tate and M. van den Bergh, Some algebras associated to automorphisms of elliptic curves, The Grothendieck Festschrift (P. Cartier et al., eds.), Birkhäuser, Boston, 1990, Vol. 1, pp. 33-85. MR 92e: 14002

[ATV2] M. Artin, J. Tate and M. van den Bergh, Modules over regular algebras of dimension 3, Invent Math. 106 (1991), 335-388. MR 93e:16055

[Be] G. M. Bergman, A note on growth functions of algebras and semigroups, mimeographed notes, University of California, Berkeley, 1978.

[BK] W. Borho and H. Kraft, Über die Gelfand-Kirillov-Dimension, Math. Annalen 220 (1976), 1-24. MR 54:367

[Co] P. M. Cohn, Skew field constructions, London Math. Soc. Lec. Notes Series 27, Cambridge University Press, 1977. MR 57:3190

[GK] I. M. Gelfand and A. A. Kirillov, Sur les corps liés aux algèbres enveloppantes des algèbres de Lie, Publ. Math. IHES 31 (1966), 5-19. MR 34:7731

[GZ] A. Giaquinto and J. J. Zhang, Quantum Weyl algebras, J. Alg. 176 (1995), 861-881. CMP 96:1

[Ja] A. V. Jategaonkar, Ore domains and free algebras, Bull. London Math. Soc. 1 (1969), 45-46. MR 39:241

[Ji] M. Jimbo, A q-difference analogue of $U(\mathfrak{g})$ and the Yang-Baxter equation, Lett. Math. Phys. 10 (1985), 63-69. MR 86k:17008

[JZ] N. Jing and J. J. Zhang, Quantum Weyl algebras and deformations of $U(\mathfrak{g})$, Pacific J. Math. (in press).

[Jo] A. Joseph, Gelfand-Kirillov dimension for algebras associated with the Weyl algebra, Ann. Inst. H. Poincaré Sect. A (N.S.) 17 (1972), 325-336. MR 49:2868

[KL] G. Krause and T. H. Lenagan, Growth of algebras and Gelfand-Kirillov dimension, Research Notes in Mathematics, Pitman Adv. Publ. Program, vol 116 (1985). MR 86g:16001

[LMO] A. Leroy, J. Matczuk and J. Okninski, On the Gelfand-Kirillov dimension of normal localizations and twisted polynomial rings, Perspectives in Ring Theory (F. van Oystaeyen and L. Le Bruyn, eds.), Kluwer Academic Publishers, 1988, pp. 205-214. MR 91c:16020

[Le] T. Levasseur, Some properties of non-commutative regular graded rings, Glasgow J. Math. 34 (1992), 277-300. MR 93k:16045

[Lo] M. Lorenz, On the transcendence degree of group algebras of nilpotent groups, Glasgow Math. J. 25 (1984), 167-174. MR 86c: 16005

[M-L] L. Makar-Limanov, The skew field of fractions of the Weyl algebra contains a free noncommutative subalgebra, Comm. Algebra 11 (1983), 2003-2006. MR 84j:16012

[Ma] W. S. Martindale III, On semiprime PI rings, Proc. Amer. Math. Soc. 40 (1973), no. 2, 365-369. MR 47:6762

[MR] J. C. McConnell and J. C. Robson, Noncommutative Noetherian Rings, Wiley-Interscience, Chichester, 1987. MR 89j:16023

[NV1] C. Năstăsescu and F. Van Oystaeyen, Graded Ring Theory, North Holland, Amsterdam, 1982. MR 84i: 16002

[NV2] C. Năstăsescu and F. Van Oystaeyen, Dimensions of Ring Theory, D. Reidel Publishing Company, 1987. MR 88f: 16029

[Pa] D. S. Passman, The Algebraic Structure of Group Rings, Wiley-Interscience, 1977. MR 81d: 16001

[Sc] A. H. Schofield, Questions on skew fields, Methods in Ring Theory (F. van Oystaeyen, ed.), D. Reidel Publishing Company, 1984, pp. 489-495. MR 86f:16017 
[SSW] L. W. Small, J. T. Stafford and R. B. Warfield, Affine algebras of Gelfand-Kirillov dimension one are PI, Math. Proc. Camb. Phil. Soc. 97 (1985), 407-414. MR 86g:16025

[SSta] S. P. Smith and J. T. Stafford, Regularity of the four dimensional Sklyanin algebra, Compositio Math. 83 (1992), 259-289. MR 93h:16037

[Su] A. Sudbery, Consistent multiparameter quantisation of $G L(n)$, J. Phys., A 23 (1990), L697-704. MR 91m:17022

[Z1] J. J. Zhang, Twisted graded algebras and equivalences of graded categories, Proc. London Math. Soc. (in press).

[Z2] J. J. Zhang, On lower transcendence degree, in preparation.

Department of Mathematics, Box 354350, University of Washington, Seattle, WashINGTON 98195

E-mail address: Zhang@math.washington.edu 